\title{
Checkpointing algorithms and fault prediction
}

\author{
Guillaume Aupy ${ }^{1,4}, Y_{\text {ves Robert }}^{1,3,4}$, Frédéric Vivien ${ }^{2,4}$ and Dounia Zaidouni ${ }^{2,4}$ \\ 1. École Normale Supérieure de Lyon \\ 2. INRIA, France \\ 3. University of Tennessee Knoxville, USA \\ 4. LIP - Université de Lyon - CNRS : UMR5668 - INRIA - \\ École Normale Supérieure de Lyon- Université Claude Bernard - Lyon, France \\ \{Guillaume.Aupy|Yves.Robert|Frederic.Vivien|Dounia.Zaidouni\}@ens-lyon.fr
}

February 28, 2022

\begin{abstract}
This paper deals with the impact of fault prediction techniques on checkpointing strategies. We extend the classical first-order analysis of Young and Daly in the presence of a fault prediction system, characterized by its recall and its precision. In this framework, we provide optimal algorithms to decide whether and when to take predictions into account, and we derive the optimal value of the checkpointing period. These results allow to analytically assess the key parameters that impact the performance of fault predictors at very large scale.
\end{abstract}

\section{Introduction}

Nowadays, the most powerful High Performance Computing systems experience about one fault per day [1, 2]. Consider the relative slopes describing the evolution of the reliability of individual components on one side, and the evolution of the number of components on the other side: the reliability of an entire platform is expected to decrease, due to probabilistic amplification, as its number of components increases. Therefore, applications running on large computing systems have to cope with platform faults. There are two main approaches. On the one hand, applications can use fault-tolerance mechanisms such as checkpoint and rollback in order to become resilient. On the other hand, system administrators can try to predict where and when faults will strike. Although considerable research has been devoted to fault predictors $[3,4,5,6,7,8$, , no predictor will ever be able to predict every fault. Therefore, fault predictors will have to be used in conjunction with fault-tolerance mechanisms.

In this paper, we assess the impact of fault prediction techniques on checkpointing strategies. We assume to have jobs executing on a platform subject to faults, and we let $\mu$ be the Mean Time Between Faults (MTBF) of the platform. In the absence of fault prediction, the standard approach is to take periodic checkpoints, each of length $C$, every period of duration $T$. In steady-state utilization of the platform, the value $T_{\mathrm{opt}}$ of $T$ that minimizes the expected waste of resource usage due to checkpointing is approximated as $T_{\mathrm{opt}}=\sqrt{2 \mu C}+C$, or $T_{\mathrm{opt}}=\sqrt{2(\mu+R) C}+C$ (where $R$ is the duration of the recovery). The former expression is the well-known Young's formula [9], while the latter is due to Daly [10].

Now, when some fault prediction mechanism is available, can we compute a better checkpointing period to decrease the expected waste? and to what extent? Critical parameters that characterize a fault prediction system are its recall $r$, which is the fraction of faults that are indeed predicted, and its precision $p$, which is the fraction of predictions that are correct (i.e., correspond to actual faults). The major objective of this paper is to refine the expression of the expected waste as a function of these new parameters, and to design efficient checkpointing policies that take predictions into account. The key contributions of this paper are:

- A refined first-order analysis in the absence of fault prediction. It leads to similar performance to Young [9] and Daly [10] when faults follow an Exponential distribution, and to better performance when faults follow a Weibull distribution. 
- The extension of this analysis to fault predictions, and the design of new checkpointing policies that takes optimal decisions on whether and when to take these predictions into account (or to ignore them).

- For policies where the decision to trust the predictor is taken with the same probability throughout the checkpointing period, we show that we should always trust the predictor, or never, depending upon platform and predictor parameters.

- For policies where the decision to trust the predictor is taken with variable probability during the checkpointing period, we show that we should change strategy only once in the period, moving from never trusting the predictor when the prediction arrives in the beginning of the period, to always trusting the predictor when the prediction arrives later on in the period, and we determine the optimal break-even point.

- For all policies, we compute the optimal value of the checkpointing period thereby designing optimal algorithms to minimize the waste when coupling checkpointing with predictions.

- An extensive set of simulations that corroborates all mathematical derivations. These simulations are based on synthetic fault traces (for Exponential fault distributions, and for more realistic Weibull fault distributions) and on log-based fault traces. In addition, they include exact prediction dates and uncertainty intervals for these dates.

The rest of the paper is organized as follows. We first detail the framework in Section 2 , We revisit Young and Daly's approach in Section 3 . We provide optimal algorithms to account for predictions in Section 4 4 we start with simpler policies where the decision to trust the predictor is taken with the same probability throughout the checkpointing period (Section 4.1) before dealing with the most general approach where the decision to trust the predictor is taken with variable probability during the checkpointing period (Section 4.2). Section 5 is devoted to simulations: we first describe the framework (Section 5.1) and then discuss synthetic and log-based failure traces in Sections 5.2 and 5.3 respectively. We discuss related work in Section 6. Finally, we provide concluding remarks in Section 7 .

$p \quad$ Predictor precision: proportion of true positives among the number of predicted faults

$r$ Predictor recall: proportion of predicted faults among total number of faults

$q$ Probability to trust the predictor

MTBF Mean Time Between Faults

$N \quad$ Number of processors in the platform

$\mu \quad$ Platform MTBF

$\mu_{\text {ind }}$ Individual MTBF

$\mu_{\mathrm{P}} \quad$ Rate of predicted faults

$\mu_{\mathrm{NP}} \quad$ Rate of unpredicted faults

$\mu_{\mathrm{e}} \quad$ Rate of events (predictions or unpredicted faults)

$D$ Downtime

$R$ Recovery time

$C$ Duration of a regular checkpoint

$C_{p} \quad$ Duration of a proactive checkpoint

$T$ Duration of a period

Table 1: Table of main notations.

\section{Framework}

\subsection{Checkpointing strategy}

We consider a platform subject to faults. Our work is agnostic of the granularity of the platform, which may consist either of a single processor, or of several processors that work concurrently and use coordinated checkpointing. Checkpoints are taken at regular intervals, or periods, of length $T$. We denote by $C$ the duration of a checkpoint (all checkpoints have same duration). By construction, we must enforce that $C \leq T$. When a fault strikes the platform, the application is lacking some resource for a certain period of time of length $D$, the downtime. The downtime accounts for software rejuvenation 
(i.e., rebooting [11, 12]) or for the replacement of the failed hardware component by a spare one. Then, the application recovers from the last checkpoint. $R$ denotes the duration of this recovery time.

\section{$2.2 \quad$ Fault predictor}

A fault predictor is a mechanism that is able to predict that some faults will take place, either at a certain point in time, or within some time-interval window. In this paper, we assume that the predictor is able to provide exact prediction dates, and to generate such predictions early enough so that a proactive checkpoint can indeed be taken before the event.

The accuracy of the fault predictor is characterized by two quantities, the recall and the precision. The recall $r$ is the fraction of faults that are predicted while the precision $p$ is the fraction of fault predictions that are correct. Traditionally, one defines three types of events: (i) True positive events are faults that the predictor has been able to predict (let True $_{P}$ be their number); (ii) False positive events are fault predictions that did not materialize as actual faults (let False P $_{P}$ be their number); and (iii) False negative events are faults that were not predicted (let False ${ }_{N}$ be their number). With these definitions, we have $r=\frac{\text { True }_{P}}{\text { True }_{P}+\text { Fals }_{N}}$ and $p=\frac{\text { True }_{P}}{\text { True }_{P}+\text { False }_{P}}$.

Proactive checkpoints may have a different length $C_{p}$ than regular checkpoints of length $C$. In fact there are many scenarios. On the one hand, we may well have $C_{p}>C$ in scenarios where regular checkpoints are taken at time-steps where the application memory footprint is minimal [13]; on the contrary, proactive checkpoints are taken according to predictions that can take place at arbitrary instants. On the other hand, we may have $C_{p}<C$ in other scenarios [8], e.g., when the prediction is localized to a particular resource subset, hence allowing for a smaller volume of checkpointed data.

To keep full generality, we deal with two checkpoint sizes in this paper: $C$ for periodic checkpoints, and $C_{p}$ for proactive checkpoints (those taken upon predictions).

In the literature, the lead time is the interval between the date at which the prediction is made available, and the actual prediction date. While the lead time is an important parameter, the shape of its distribution law is irrelevant to the problem: either a fault is predicted at least $C_{p}$ seconds in advance, and then one can checkpoint just in time before the fault, or the prediction is useless! In other words, predictions that come too late should be classified as unpredicted faults whenever they materialize as actual faults, leading to a smaller value of the predictor recall.

\section{$2.3 \quad$ Fault rates}

The key parameter is $\mu$, the MTBF of the platform. If the platform is made of $N$ components whose individual MTBF is $\mu_{\mathrm{ind}}$, then $\mu=\frac{\mu_{\mathrm{ind}}}{N}$. This result is true regardless of the fault distribution law ${ }^{1}$,

In addition to $\mu$, the platform $\mathrm{MTBF}$, let $\mu_{\mathrm{P}}$ be the mean time between predicted events (both true positive and false positive), and let $\mu_{\mathrm{NP}}$ be the mean time between unpredicted faults (false negative). Finally, we define the mean time between events as $\mu_{\mathrm{e}}$ (including all three event types). The relationships between $\mu, \mu_{\mathrm{P}}, \mu_{\mathrm{NP}}$, and $\mu_{\mathrm{e}}$ are the following:

- Rate of unpredicted faults: $\frac{1}{\mu_{\mathrm{NP}}}=\frac{1-r}{\mu}$, since $1-r$ is the fraction of faults that are unpredicted;

- Rate of predicted faults: $\frac{r}{\mu}=\frac{p}{\mu_{\mathrm{P}}}$, since $r$ is the fraction of faults that are predicted, and $p$ is the fraction of fault predictions that are correct;

- Rate of events: $\frac{1}{\mu_{\mathrm{e}}}=\frac{1}{\mu_{\mathrm{P}}}+\frac{1}{\mu_{\mathrm{NP}}}$, since events are either predictions (true or false), or unpredicted faults.

\subsection{Objective: waste minimization}

The natural objective is to minimize the expectation of the total execution time, makespan, of the application. Instead, in order to ease mathematical derivations, we aim at minimizing the waste. The waste is the expected percentage of time lost, or "wasted", during the execution. In other words, the waste is the fraction of time during which the platform is not doing useful work. This definition was introduced by Wingstrom [14. Obviously, the lower the waste, the lower the expected makespan, and

\footnotetext{
${ }^{1}$ For the sake of completeness, we provide a proof of this widely-used result in $\mathrm{A}$. To the best of our knowledge, no proof has been published in the literature yet.
} 
reciprocally. Hence the two objectives are strongly related and minimizing one of them also minimizes the other.

\section{Revisiting Daly's first-order approximation}

Young proposed in [9] a "first order approximation to the optimum checkpoint interval". Young's formula was later refined by Daly [10] to take into account the recovery time. We revisit their analysis using the notion of waste.

Let TIME $E_{\text {base }}$ be the base time of the application without any overhead (neither checkpoints nor faults). First, assume a fault-free execution of the application with periodic checkpointing. In such an environment, during each period of length $T$ we take a checkpoint, which lasts for a time $C$, and only $T-C$ units of work are executed. Let Time $\mathrm{FF}_{\mathrm{F}}$ be the execution time of the application in this setting. Following most works in the literature, we also take a checkpoint at the end of the execution. The fault-free execution time $\mathrm{TIME}_{\mathrm{FF}}$ is equal to the time needed to execute the whole application, TimE $\mathrm{b}_{\mathrm{base}}$, plus the time taken by the checkpoints:

$$
\text { TIMEFF }_{\mathrm{F}}=\mathrm{TIME}_{\mathrm{base}}+N_{\mathrm{ckpt}} C
$$

where $N_{\text {ckpt }}$ is the number of checkpoints taken. We have

$$
N_{\text {ckpt }}=\left\lceil\frac{\text { TIME }_{\text {base }}}{T-C}\right\rceil \approx \frac{\text { TIME }_{\text {base }}}{T-C}
$$

When discarding the ceiling function, we assume that the execution time is very large with respect to the period or, symmetrically, that there are many periods during the execution. Plugging back the (approximated) value $N_{\mathrm{ckpt}}=\frac{\text { TIME }_{\mathrm{base}}}{T-C}$, we derive that

$$
\operatorname{TiMEF}_{\mathrm{FF}}=\frac{\text { TimE }_{\text {base }}}{T-C} T
$$

The waste due to checkpointing in a fault-free execution, WASTEFF, is defined as the fraction of the execution time that does not contribute to the progress of the application:

$$
\text { WASTE }_{\mathrm{FF}}=\frac{\text { TIMEFF }_{\mathrm{F}}-\text { TIME }_{\text {base }}}{\text { TIMEFF }_{\mathrm{F}}} \Leftrightarrow \quad\left(1-\text { WASTEFF }_{\mathrm{F}}\right) \mathrm{TIME}_{\mathrm{FF}}=\text { TIME }_{\mathrm{base}}
$$

Combining Equations (2) and (3), we get:

$$
\mathrm{WASTE}_{\mathrm{FF}}=\frac{C}{T}
$$

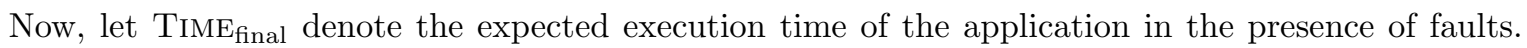
This execution time can be divided into two parts: (i) the execution of "chunks" of work of size $T-C$ followed by their checkpoint; and (ii) the time lost due to the faults. This decomposition is illustrated by Figure 1. The first part of the execution time is equal to TimefF. Let $N_{\text {faults }}$ be the number of faults occurring during the execution, and let $T_{\text {lost }}$ be the average time lost per fault. Then,

$$
\mathrm{TIME}_{\text {final }}=\mathrm{TIME}_{\mathrm{FF}}+N_{\text {faults }} \times T_{\text {lost }}
$$

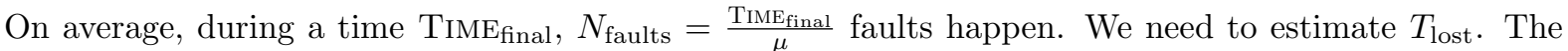
instants at which periods begin and at which faults strike are independent. Therefore, the expected time elapsed between the completion of the last checkpoint and a fault is $\frac{T}{2}$ for all distribution laws, regardless of their particular shape. We conclude that $T_{\text {lost }}=\frac{T}{2}+D+R$, because after each fault there is a downtime and a recovery. This leads to:

$$
\mathrm{TIME}_{\mathrm{final}}=\mathrm{TIME}_{\mathrm{FF}}+\frac{\text { TIME }_{\text {final }}}{\mu} \times\left(D+R+\frac{T}{2}\right)
$$

Let WASTE $\mathrm{fault}_{\mathrm{f}}$ be the fraction of the total execution time that is lost because of faults:

$$
\text { WASTE }_{\text {fault }}=\frac{\text { TIME }_{\text {final }}-\text { TIMEFF }_{\text {Final }}}{\text { TIME }_{\text {final }}} \Leftrightarrow \quad\left(1-\text { WASTE }_{\text {fault }}\right) \operatorname{TIME}_{\text {final }}=\operatorname{TIME}_{\mathrm{FF}}
$$


We derive:

$$
\text { WASTE}_{\text {fault }}=\frac{1}{\mu}\left(D+R+\frac{T}{2}\right)
$$

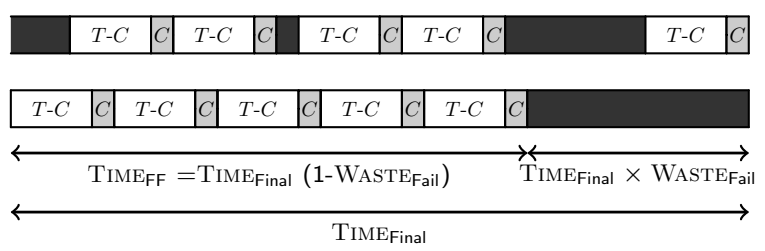

Figure 1: An execution (top), and its re-ordering (bottom), to illustrate both sources of waste. Blackened intervals correspond to work destroyed by faults, downtimes, and recoveries.

In [10, Daly uses the expression

$$
\operatorname{TIME}_{\text {final }}=\left(1+\text { WASTE}_{\text {fault }}\right) \operatorname{TIME}_{\mathrm{FF}}
$$

instead of Equation (6), which leads him to his well-known first-order formula

$$
T=\sqrt{2(\mu+(D+R)) C}+C
$$

Figure 1 explains why Equation (8) is not correct and should be replaced by Equation (6). Indeed, the expected number of faults depends on the final time, not on the time for a fault-free execution. We point out that Young 9] also used Equation (8), but with $D=R=0$. Equation (6) can be rewritten $\mathrm{TIME}_{\mathrm{final}}=\mathrm{TIME}_{\mathrm{FF}} /\left(1-\mathrm{WASTE}_{\mathrm{fault}}\right)$. Therefore, using Equation (8) instead of Equation (6), in fact, is equivalent to write $\frac{1}{1-\text { WASTE }_{\text {fault }}} \approx 1+$ WASTE $_{\text {fault }}$ which is indeed a first-order approximation if WASTE fault $\ll 1$.

Now, let Waste denote the total waste:

$$
\text { WAStE }=\frac{\text { TIME }_{\text {final }}-\text { TIME }_{\text {base }}}{\text { TimE }_{\text {final }}}
$$

Therefore

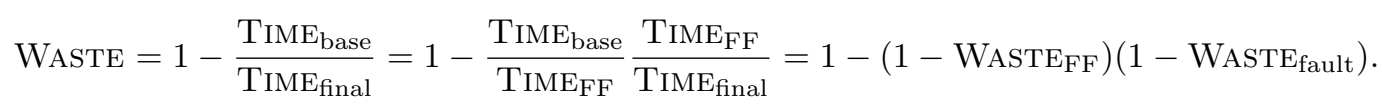

Altogether, we derive the final result:

$$
\begin{aligned}
\text { WASTE } & =\text { WASTE }_{\mathrm{FF}}+\text { WASTE }_{\mathrm{fault}}-\text { WASTE }_{\mathrm{FF}} \text { WASTE }_{\mathrm{fault}} \\
& =\frac{C}{T}+\left(1-\frac{C}{T}\right) \frac{1}{\mu}\left(D+R+\frac{T}{2}\right)
\end{aligned}
$$

We obtain WASte $=\frac{u}{T}+v+w T$ where $u=C\left(1-\frac{D+R}{\mu}\right), v=\frac{D+R-C / 2}{\mu}$, and $w=\frac{1}{2 \mu}$. Thus WASte is minimized for $T=\sqrt{\frac{u}{w}}$. The Refined First-Order (RFO) formula for the optimal period is thus:

$$
T_{\mathrm{RFO}}=\sqrt{2(\mu-(D+R)) C}
$$

It is interesting to point out why Equation (13) is a first-order approximation, even for large jobs. Indeed, there are several restrictions to enforce for the approach to be valid:

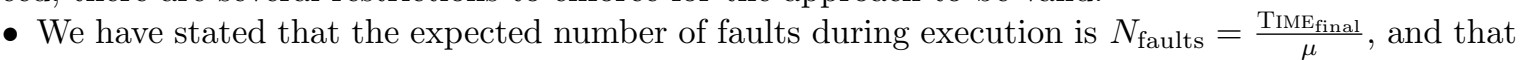
the expected time lost due to a fault is $T_{\text {lost }}=\frac{T}{2}$. Both statements are true individually, but the expectation of a product is the product of the expectations only if the random variables are

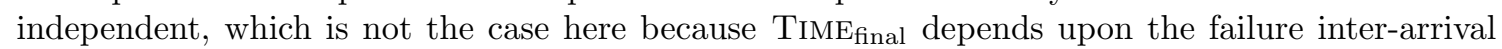
times.

- In Equation (4), we have to enforce $C \leq T$ to have WASTE $_{\mathrm{FF}} \leq 1$ 
- In Equation (7), we have to enforce $D+R \leq \mu$ and to bound $T$ in order to have WASTEfault $\leq 1$. Intuitively, we need $\mu$ to be large enough for Equation (7) to make sense. However, regardless of the value of the individual MTBF $\mu_{\text {ind }}$, there is always a threshold in the number of components $N$ above which the platform MTBF $\mu=\frac{\mu_{\text {ind }}}{N}$ becomes too small for Equation (7) to be valid.

- Equation (7) is accurate only when two or more faults do not take place within the same period. Although unlikely when $\mu$ is large in front of $T$, the possible occurrence of many faults during the same period cannot be eliminated.

To ensure that the latter condition (at most a single fault per period) is met with a high probability, we cap the length of the period: we enforce the condition $T \leq \alpha \mu$, where $\alpha$ is some tuning parameter chosen as follows. The number of faults during a period of length $T$ can be modeled as a Poisson process of parameter $\beta=\frac{T}{\mu}$. The probability of having $k \geq 0$ faults is $P(X=k)=\frac{\beta^{k}}{k !} e^{-\beta}$, where $X$ is the number of faults. Hence the probability of having two or more faults is $\pi=P(X \geq 2)=$ $1-(P(X=0)+P(X=1))=1-(1+\beta) e^{-\beta}$. If we assume $\alpha=0.27$ then $\pi \leq 0.03$, hence a valid approximation when bounding the period range accordingly. Indeed, with such a conservative value for $\alpha$, we have overlapping faults for only $3 \%$ of the checkpointing segments in average, so that the model is quite reliable. For consistency, we also enforce the same type of bound on the checkpoint time, and on the downtime and recovery: $C \leq \alpha \mu$ and $D+R \leq \alpha \mu$. However, enforcing these constraints may lead to use a sub-optimal period: it may well be the case that the optimal period $\sqrt{2(\mu-(D+R)) C}$ of Equation (13) does not belong to the admissible interval $[C, \alpha \mu]$. In that case, the waste is minimized for one of the bounds of the admissible interval: this is because, as seen from Equation (12), the waste is a convex function of the period.

We conclude this discussion on a positive note. While capping the period, and enforcing a lower bound on the MTBF, is mandatory for mathematical rigor, simulations (see Section 5 for both Exponential and Weibull distributions) show that actual job executions can always use the value from Equation (13), accounting for multiple faults whenever they occur by re-executing the work until success. The firstorder model turns out to be surprisingly robust!

To the best of our knowledge, despite all the limitations above, there is no better approach to estimate the waste due to checkpointing when dealing with arbitrary fault distributions. However, assuming that faults obey an Exponential distribution, it is possible to use the memory-less property of this distribution to provide more accurate results. A second-order approximation when faults obey an Exponential distribution is given in Daly [10, Equation (20)] as TIME $\operatorname{Tinal}=\mu e^{R / \mu}\left(e^{\frac{T}{\mu}}-1\right) \frac{\text {TIME}_{\text {base }}}{T-C}$. In fact, in that case, the exact value of TIME $\mathrm{final}_{\text {inal }}$ is provided in [15, 16] as $\operatorname{TimE}_{\mathrm{final}}=(\mu+D) e^{R / \mu}\left(e^{\frac{T}{\mu}}-1\right) \frac{\text { TIME }_{\text {base }}}{T-C}$,

and the optimal period is then $\frac{1+\mathbb{L}\left(-e^{-\frac{C}{\mu}-1}\right)}{\mu}$ where $\mathbb{L}$, the Lambert function, is defined as $\mathbb{L}(z) e^{\mathbb{L}(z)}=z$.

To assess the accuracy of the different first order approximations, we compare the periods defined by Young's formula [9, Daly's formula [10, and Equation [13), to the optimal period, in the case of an Exponential distribution. Results are reported in Table 2 To establish these results, we use the same parameters as in Section 5 $C=R=600 \mathrm{~s}, D=60 \mathrm{~s}$, and $\mu_{\text {ind }}=125$ years. Furthermore, to compute the optimal period, for each platform size we choose the application size so that TIME $_{\text {base }}=2$ hours. One can observe in Table 2 that the relative error for Daly's period is slightly larger than the one for Young's period. In turn, the absolute value of the relative error for Young's period is slightly larger than the one for RFO. More importantly, when Young's and Daly's formulas overestimate the period, RFO underestimates it. Table 2 does not allow us to assess whether these differences are actually significant. However we also report in Section 5.2 some simulations that show that Equation $(13)$ leads to smaller execution times for Weibull distributions than both classical formulas (Tables 4 and 5 .

\section{Taking predictions into accounts}

In this section, we present an analytical model to assess the impact of predictions on periodic checkpointing strategies. As already mentioned, we consider the case where the predictor is able to provide exact prediction dates, and to generate such predictions at least $C_{p}$ seconds in advance, so that a proactive checkpoint of length $C_{p}$ can indeed be taken before the event.

For the sake of clarity, we start with a simple algorithm (Section 4.1) which we refine in Section 4.2 , We then compute the value of the period that minimizes the waste in Section 4.3 


\begin{tabular}{|r|r|rr|rr|rr|r|}
\hline$N$ & \multicolumn{1}{|c|}{$\mu$} & \multicolumn{2}{|c|}{ YOUNG } & \multicolumn{2}{|c|}{ DALY } & \multicolumn{2}{|c|}{ RFO } & Optimal \\
\hline $2^{10}$ & 3849609 & 68567 & $(0.5 \%)$ & 68573 & $(0.5 \%)$ & 67961 & $(-0.4 \%)$ & 68240 \\
$2^{11}$ & 1924805 & 48660 & $(0.7 \%)$ & 48668 & $(0.7 \%)$ & 48052 & $(-0.6 \%)$ & 48320 \\
$2^{12}$ & 962402 & 34584 & $(1.2 \%)$ & 34595 & $(1.2 \%)$ & 33972 & $(-0.6 \%)$ & 34189 \\
$2^{13}$ & 481201 & 24630 & $(1.6 \%)$ & 24646 & $(1.7 \%)$ & 24014 & $(-0.9 \%)$ & 24231 \\
$2^{14}$ & 240601 & 17592 & $(2.3 \%)$ & 17615 & $(2.5 \%)$ & 16968 & $(-1.3 \%)$ & 17194 \\
$2^{15}$ & 120300 & 12615 & $(3.2 \%)$ & 12648 & $(3.5 \%)$ & 11982 & $(-1.9 \%)$ & 12218 \\
$2^{16}$ & 60150 & 9096 & $(4.5 \%)$ & 9142 & $(5.1 \%)$ & 8449 & $(-2.9 \%)$ & 8701 \\
$2^{17}$ & 30075 & 6608 & $(6.3 \%)$ & 6673 & $(7.4 \%)$ & 5941 & $(-4.4 \%)$ & 6214 \\
$2^{18}$ & 15038 & 4848 & $(8.8 \%)$ & 4940 & $(10.8 \%)$ & 4154 & $(-6.8 \%)$ & 4458 \\
$2^{19}$ & 7519 & 3604 & $(12.0 \%)$ & 3733 & $(16.0 \%)$ & 2869 & $(-10.8 \%)$ & 3218 \\
\hline
\end{tabular}

Table 2: Comparing periods produced by the different approximations with optimal value. Beside each period, we report its relative deviation to the optimal. Each value is expressed in seconds.

\subsection{Simple policy}

In this section, we consider the following algorithm:

- While no fault prediction is available, checkpoints are taken periodically with period $T$;

- When a fault is predicted, there are two cases: either there is the possibility to take a proactive checkpoint, or there is not enough time to do so, because we are already checkpointing (see Figures 2(b) and 2(c)). In the latter case, there is no other choice than ignoring the prediction. In the former case, we still have the possibility to ignore the prediction, but we may also decide to trust it: in fact the decision is randomly taken. With probability $q$, we trust the predictor and take the prediction into account (see Figures $2(\mathrm{f})$ and $2(\mathrm{~g})$ ), and with probability $1-q$, we ignore the prediction (see Figures 2(d) and 2(e));

- If we take the prediction into account, we take a proactive checkpoint (of length $C_{p}$ ) as late as possible, i.e., so that it completes right at the time when the fault is predicted to happen. After this checkpoint, we complete the execution of the period (see Figures $2(\mathrm{f})$ and 2 (g));

- If we ignore the prediction, either by necessity (not enough time to take an extra checkpoint, see Figures 2(b) and 2(c)), or or by choice (with probability $1-q$, Figures 2(d) and 2(e)), we finish the current period and start a new one.

The rationale for not always trusting the predictor is to avoid taking useless checkpoints too frequently. Intuitively, the precision $p$ of the predictor must be above a given threshold for its usage to be worthwhile. In other words, if we decide to checkpoint just before a predicted event, either we will save time by avoiding a costly re-execution if the event does correspond to an actual fault, or we will lose time by unduly performing an extra checkpoint. We need a larger proportion of the former cases, i.e., a good precision, for the predictor to be really useful. The following analysis will determine the optimal value of $q$ as a function of the parameters $C, C_{p}, \mu, r$, and $p$.

We could refine the approach by taking into account the amount of work already done in the current period when deciding whether to trust the predictor or not. Intuitively, the more work already done, the more important to save it, hence the more worthwhile to trust the predictor. We design such a refined strategy in Section 4.2. Right now, we analyze a simpler algorithm where we decide to trust or not to trust the predictor, independently of the amount of work done so far within the period.

We analyze the algorithm in order to compute a formula for the expected waste, just as in Equation (12). While the value of $\mathrm{WASTE}_{\mathrm{FF}}$ is unchanged (WASTEFF $=\frac{C}{T}$ ), the value of WASTE $\mathrm{W}_{\text {fault }}$ is modified because of predictions. As illustrated in Figure 2, there are many different scenarios that contribute to WASTEfault that can be sorted into three categories:

(1) Unpredicted faults: This overhead occurs each time an unpredicted fault strikes, that is, on average, once every $\mu_{\mathrm{NP}}$ seconds. Just as in Equation (7), the corresponding waste is $\frac{1}{\mu_{\mathrm{NP}}}\left[\frac{T}{2}+D+R\right]$. 


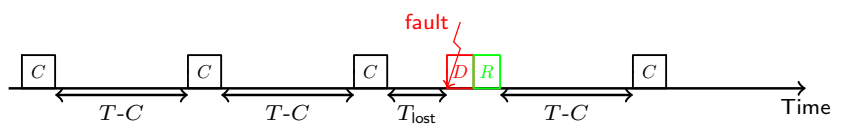

(a) Unpredicted fault

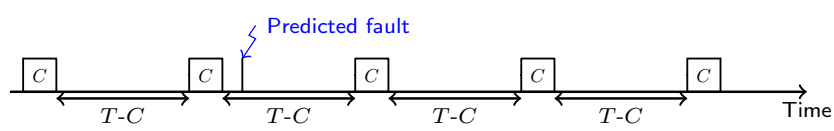

(b) Prediction cannot be taken into account - no actual fault

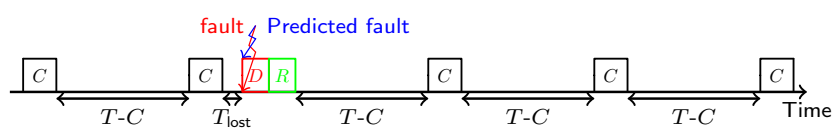

(c) Prediction cannot be taken into account - with actual fault

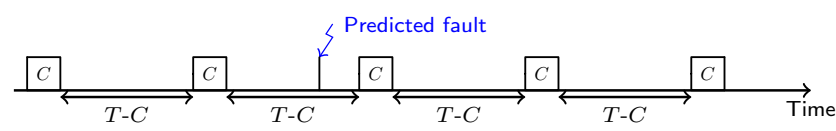

(d) Prediction not taken into account by choice - no actual fault

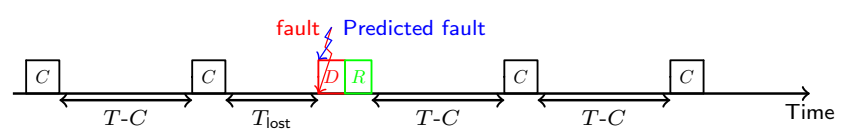

(e) Prediction not taken into account by choice - with actual fault

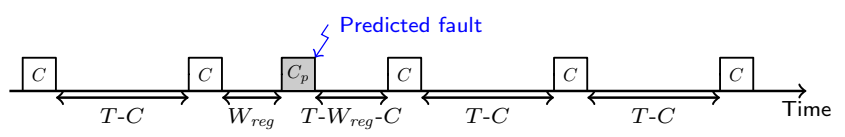

(f) Prediction taken into account - no actual fault

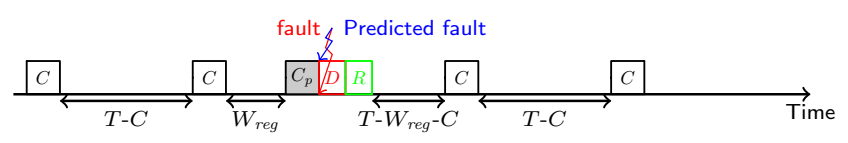

(g) Prediction taken into account - with actual fault

Figure 2: Actions taken for the different event types. 
(2) Predictions not taken into account: The second source of waste is for predictions that are ignored. This overhead occurs in two different scenarios. First, if we do not have time to take a proactive checkpoint, we have an overhead if and only the prediction is an actual fault. This case happens with probability $p$. We then lose a time $t+D+R$ if the predicted fault happens a time $t$ after the completion of the last periodic checkpoint. The expected time lost is thus

$$
T_{\text {lost }}^{1}=\frac{1}{T} \int_{0}^{C_{p}}(p(t+D+R)+(1-p) 0) d t
$$

Then, if we do have time to take a proactive checkpoint but still decide to ignore the prediction, we also have an overhead if and only the prediction is an actual fault, but the expected time lost is now weighted by the probability $(1-q)$ :

$$
T_{\text {lost }}^{2}=(1-q) \frac{1}{T} \int_{C_{p}}^{T}(p(t+D+R)+(1-p) 0) d t
$$

(3) Predictions taken into account: We now compute the overhead due to a prediction which we trust (hence we checkpoint just before its date). If the prediction is an actual fault, we lose $C_{p}+D+R$ seconds, but if it is not, we lose the unnecessary extra checkpoint time $C_{p}$. The expected time lost is now weighted by the probability $q$ and becomes

$$
T_{\text {lost }}^{3}=q \frac{1}{T} \int_{C_{p}}^{T}\left(p\left(C_{p}+D+R\right)+(1-p) C_{p}\right) d t
$$

We derive the final value of WASTE fault:

$$
\mathrm{WASTE}_{\text {fault }}=\frac{1}{\mu_{\mathrm{NP}}}\left[\frac{T}{2}+D+R\right]+\frac{1}{\mu_{\mathrm{P}}}\left[T_{\text {lost }}^{1}+T_{\text {lost }}^{2}+T_{\text {lost }}^{3}\right]
$$

This final expression comes from the disjunction of all possibles cases, using the Law of Total Probability [17, p.23]: the waste comes either from non-predicted faults or from predictions; in the latter case, we have analyzed the three possible sub-cases and weighted them with their respective probabilities. After simplifications, we obtain

$$
\mathrm{WASTE}_{\text {fault }}=\frac{1}{\mu}\left((1-r q) \frac{T}{2}+D+R+\frac{q r}{p} C_{p}-\frac{q r C_{p}^{2}}{p T}(1-p / 2)\right)
$$

We could now plug this expression back into Equation (11) to compute the value of $T$ that minimizes the total waste. Instead, we move on to describing the refined algorithm, and we minimize the waste for the refined strategy, since it always induces a smaller waste.

\subsection{Refined policy}

In this section, we refine the approach and consider different trust strategies, depending upon the time in the period where the prediction takes place. Intuitively, the later in the period, the more likely we are inclined to trust the predictor, because the amount of work that we could lose gets larger and larger. As before, we cannot take into account a fault predicted to happen less than $C_{p}$ units of time after the beginning of the period. Therefore, we focus on what happens in the period after time $C_{p}$. Formally, we now divide the interval $\left[C_{p}, T\right]$ into $n$ intervals $\left[\beta_{i} ; \beta_{i+1}\right]$ for $i \in\{0, \cdots, n-1\}$, where $\beta_{0}=C_{p}$ and $\beta_{n}=T$. For each interval $\left[\beta_{i} ; \beta_{i+1}\right]$, we trust the predictor with probability $q_{i}$. We aim at determining the values of $n, \beta_{i}$, and $q_{i}$ that minimize the waste. As mentioned before, intuition tells us that the $q_{i}$ values should be non-decreasing. We prove below a somewhat unexpected theorem: in the optimal strategy, there is either one or two different $q_{i}$ values, and these values are 0 or 1 . This means that we should never trust the predictor in the beginning of a period, and always trust it in the end of the period, without any intermediate behavior in between.

We formally express this striking result below. Let $\beta_{\text {lim }}=\frac{C_{p}}{p}$. The optimal strategy is provided by Theorem 1 below. We first prove the following proposition: 
Proposition 1. The values of $\beta_{i}$ and $q_{i}$ that minimize the waste satisfy the following conditions:

(i) For all $i$ such that $\beta_{i+1} \leq \beta_{\text {lim }}, q_{i}=0$.

(ii) For all $i$ such that $\beta_{i} \geq \beta_{\text {lim }}, q_{i}=1$.

Proof. First we compute the waste with the refined algorithm, using Equation (11). The formula for WASTE fault is similar to Equation (14) on each interval:

$$
\begin{aligned}
\text { WASte } & =\frac{C}{T}+\left(1-\frac{C}{T}\right)\left[\frac{1}{\mu_{\mathrm{NP}}}\left(\frac{T}{2}+D+R\right)\right. \\
& +\frac{1}{\mu_{\mathrm{P}}} \sum_{i=0}^{n-1}\left(q_{i} \int_{\beta_{i}}^{\beta_{i+1}} \frac{\left(p\left(C_{p}+D+R\right)+(1-p) C_{p}\right)}{T} d t\right. \\
& \left.\left.+\left(1-q_{i}\right) \int_{\beta_{i}}^{\beta_{i+1}} \frac{p(t+D+R)}{T} d t\right)\right]
\end{aligned}
$$

Now, consider a fixed value of $i$ and express the value of WASTE as a function of $q_{i}$ :

$$
\text { WASTE }=K+\left(1-\frac{C}{T}\right) \frac{q_{i}}{\mu_{\mathrm{P}}} \int_{\beta_{i}}^{\beta_{i+1}}\left(\frac{C_{p}}{T}-\frac{p t}{T}\right) d t
$$

where $K$ does not depend on $q_{i}$. From the sign of the function to be integrated, one sees that WASTE is minimized when $q_{i}=0$ if $\beta_{i+1} \leq \beta_{\lim }=\frac{C_{p}}{p}$, and when $q_{i}=1$ if $\beta_{i} \geq \beta_{\lim }$.

Theorem 1. The optimal algorithm takes proactive actions if and only if the prediction falls in the interval $\left[\beta_{\text {lim }}, T\right]$.

Proof. From Proposition 1 the values for $q_{i}$ are optimally defined for every $i$ but one: we do not know the optimal value if there exists $i_{0}$ such that $\beta_{i_{0}}<\beta_{\lim }<\beta_{i_{0}+1}$. Then let us consider the waste where $q_{i_{0}}$ is replaced by $q_{i_{0}}^{(1)}$ on $\left[\beta_{i_{0}}, \beta_{\lim }\right]$ and by $q_{i_{0}}^{(2)}$ on $\left[\beta_{\lim }, \beta_{i_{0}+1}\right]$. The new waste is necessarily smaller than the one with only $q_{i_{0}}$, since we relaxed the constraint. We know from Proposition 1 that the optimal solution is then to have $q_{i_{0}}^{(1)}=0$ and $q_{i_{0}}^{(2)}=1$.

Let us now compute the value of the waste with the optimal algorithm. There are two cases, depending upon whether $T \leq \beta_{\lim }$ or not. For values of $T$ smaller than $\beta_{\lim }$, Theorem 1 shows that the optimal algorithm never takes any proactive action; in that case the waste is given by Equation 12 in Section 3 For values of $T$ larger than $\beta_{\lim }=\frac{C_{p}}{p}$, we compute the waste due to predictions as

$$
\begin{aligned}
& \frac{1}{\mu_{\mathrm{P}}} \frac{1}{T}\left(\int_{0}^{C_{p} / p} p(t+D+R) d t+\int_{C_{p} / p}^{T}\left(p\left(C_{p}+D+R\right)+(1-p) C_{p}\right) d t\right) \\
& =\frac{r}{p \mu}\left(p(D+R)+C_{p}-\frac{C_{p}^{2}}{2 p T}\right)
\end{aligned}
$$

Indeed, in accordance with Theorem 1 no prediction is taken into account in the interval $\left[0, \frac{C_{p}}{p}\right]$, while all predictions are taken into account in the interval $\left[\frac{C_{p}}{p}, T\right]$. Adding the waste due to unpredicted faults, namely $\frac{1}{\mu_{\mathrm{NP}}}\left[\frac{T}{2}+D+R\right]$, we derive

$$
\text { WASTE }_{\text {fault }}=\frac{1}{\mu}\left((1-r) \frac{T}{2}+\frac{r}{p} C_{p}\left(1-\frac{1}{2 p} \frac{C_{p}}{T}\right)+D+R\right) .
$$


Plugging this value into Equation (11), we obtain the total waste when $\frac{C_{p}}{p} \leq T$ :

$$
\begin{aligned}
\text { WASTE }= & \frac{C}{T}+\frac{1}{\mu}\left((1-r) \frac{T}{2}+\frac{r}{p} C_{p}\left(1-\frac{1}{2 p} \frac{C_{p}}{T}\right)+D+R\right)\left(1-\frac{C}{T}\right) \\
= & \frac{r C C_{p}^{2}}{2 p^{2}} \frac{1}{\mu T^{2}}+\left(\mu C-\frac{r C_{p}^{2}}{2 p^{2}}-C\left(\frac{r C_{p}}{p}+D+R\right)\right) \frac{1}{\mu T}+\frac{1-r}{2 \mu} T \\
& +\frac{-(1-r) \frac{C}{2}+\frac{r C_{p}}{p}+D+R}{\mu}
\end{aligned}
$$

Altogether, the expression for the total waste becomes:

$$
\begin{cases}\operatorname{WAStE}_{1}(T)=\frac{C\left(1-\frac{D+R}{\mu}\right)}{T}+\frac{D+R-C / 2}{\mu}+\frac{1}{2 \mu} T & \text { if } \frac{C_{p}}{p} \geq T \\ \operatorname{WAStE}_{2}(T)=\frac{r C C_{p}^{2}}{2 \mu p^{2}} \frac{1}{T^{2}}+\frac{\left(C\left(1-\frac{\frac{r C_{p}}{p}+D+R}{\mu}\right)-\frac{r C_{p}^{2}}{2 \mu p^{2}}\right)}{T}+\frac{-(1-r) \frac{C}{2}+\frac{r C_{p}}{p}+D+R}{\mu}+\frac{1-r}{2 \mu} T & \text { if } \frac{C_{p}}{p} \leq T\end{cases}
$$

One can check that when $r=0$ (no error predicted, hence no proactive action in the algorithm), then $\mathrm{WASTE}_{1}$ and $\mathrm{WASTE}_{2}$ coincide. We also check that both values coincide for $T=\frac{C_{p}}{p}$. We show how to minimize the waste in Equation (15) in Section 4.3

\subsection{Waste minimization}

In this section we focus on minimizing the waste in Equation 15. Recall that, by construction, we always have to enforce the constraint $T \geq C$. First consider the case where $C \leq \frac{C_{p}}{p}$. On the interval $T \in\left[C, \frac{C_{p}}{p}\right]$, we retrieve the optimal value found in Section 3 and derive that $\mathrm{WASTE}_{1}$, the waste when predictions are not taken into account, is minimized for

$$
T_{\mathrm{NoPRED}}=\max \left(C, \min \left(T_{\mathrm{RFO}}, \frac{C_{p}}{p}\right)\right)
$$

Indeed, the optimal value should belong to the interval $\left[C, \frac{C_{p}}{p}\right]$, and the function WAstE ${ }_{1}$ is convex: if the extremal solution $\sqrt{2(\mu-(D+R)) C}$ does not belong to this interval, then the optimal value is one of the bounds of the interval.

On the interval $T \in\left[\frac{C_{p}}{p},+\infty\right)$, we find the optimal solution by differentiating twice WASTE With $^{2}$ respect to $T$. Writing $\operatorname{WASTE}_{2}(T)=\frac{u}{T^{2}}+\frac{v}{T}+w+x T$ for simplicity, we obtain $\operatorname{WASTE}_{2}^{\prime \prime}(T)=\frac{2}{T^{3}}\left(\frac{3 u}{T}+v\right)$. Here, a key parameter is the sign of :

$$
v=\left(C\left(1-\frac{\frac{r C_{p}}{p}+D+R}{\mu}\right)-\frac{r C_{p}^{2}}{2 \mu p^{2}}\right)
$$

We detail the case $v \geq 0$ in the following, because it is the most frequent with realistic parameter sets; we do have $v \geq 0$ for all the whole range of simulations in Section 5. For the sake of completeness, we will briefly discuss the case $v<0$ in the comments below.

When $v \geq 0$, we have $\operatorname{WASTE}_{2}^{\prime \prime}(T) \geq 0$, so that $\mathrm{WASTE}_{2}$ is convex on the interval $\left[\frac{C_{p}}{p},+\infty\right)$ and admits a unique minimum $T_{\text {extr. }}$. Note that $T_{\text {extr }}$ can be computed either numerically or using Cardano's method, since it is the unique real root of a polynomial of degree 3 . The optimal solution on $\left[\frac{C_{p}}{p},+\infty\right)$ is then: $T_{\mathrm{PRED}}=\max \left(T_{\mathrm{extr}}, \frac{C_{p}}{p}\right)$.

It remains to consider the case where $\frac{C_{p}}{p}<C$. In fact, it suffices to add the constraint that the value of $T_{\mathrm{PRED}}$ should be greater than $C$, that is:

$$
T_{\mathrm{PRED}}=\max \left(C, \max \left(T_{\mathrm{extr}}, \frac{C_{p}}{p}\right)\right)
$$


Finally, the optimal solution for the waste is given by the minimum of the following two values:

$$
\left\{\begin{array}{l}
\frac{C\left(1-\frac{D+R}{\mu}\right)}{T_{\mathrm{NOPRE}}}+\frac{D+R-C / 2}{\mu}+\frac{1}{2 \mu} T_{\mathrm{NOPRED}} \\
\frac{r C C_{p}^{2}}{2 \mu p^{2}} \frac{1}{T_{\mathrm{PRED}}^{2}}+\frac{\left(C\left(1-\frac{\frac{r C_{p}}{p}+D+R}{\mu}\right)-\frac{r C_{p}^{2}}{2 \mu p^{2}}\right)}{T_{\mathrm{PRED}}}+\frac{-(1-r) \frac{C}{2}+\frac{r C_{p}}{p}+D+R}{\mu}+\frac{1-r}{2 \mu} T_{\mathrm{PRED}}
\end{array}\right.
$$

We make a few observations:

- Just as for Equation 13 in Section 3 , mathematical rigor calls for capping the values of $D, R, C$, $C_{p}$ and $T$ in front of the MTBF. The only difference is that we should replace $\mu$ by $\mu_{\mathrm{e}}$ : this is to account for the occurrence rate of all events, be they unpredicted faults or predictions.

- While the expression of the waste looks complicated, the numerical value of the optimal period can easily be computed in all cases. We have dealt with the case $v \geq 0$, where $v$ is the coefficient of $1 / T$ in $\operatorname{WASTE}_{2}(T)=\frac{u}{T^{2}}+\frac{v}{T}+w+x T$. When $v<0$ we only needs to compute all the nonnegative real roots of a polynomial of degree 3 , and check which one leads to the best value. More precisely, these root(s) partition the admissible interval $\left[\frac{C_{p}}{p},+\infty\right)$ into several sub-intervals, and the optimal value is either a root or a sub-interval bound.

- In many practical situations, when $\mu$ is large enough, we can dramatically simplify the expression of $\mathrm{WASTE}_{2}(T)$ : we have $T=O(\sqrt{\mu})$, the term $\frac{u}{T^{2}}$ becomes negligible, checkpoint parameters become negligible in front of $\mu$, and we derive the approximated value $\sqrt{\frac{2 \mu C}{1-r}}$. This value can be seen as an extension of Equation (13) giving $T_{\mathrm{RFO}}$, where $\mu$ is replaced by $\frac{\mu}{1-r}$ : faults are replaced by non-predicted faults, and the overhead due to false predictions is negligible. As a word of caution, recall that this conclusion is valid only when $\mu$ is very large in front of all other parameters.

\section{Simulation results}

We start by presenting the simulation framework (Section 5.1). Then we report results using synthetic traces (Section 5.2 and log-based traces (Section 5.3). Finally, we assess the respective impact of the two key parameters of a predictor, its recall and its precision, on checkpointing strategies (Section 5.4).

\subsection{Simulation framework}

Scenario generation - In order to check the accuracy of our model and of our analysis, and to assess the potential benefits of predictors, we study the performance of our new solutions and of pre-existing ones using a discrete-event simulator. The simulation engine generates a random trace of faults. Given a set of $p$ processors, a failure trace is a set of failure dates for each processor over a fixed time horizon $h$ (set to 2 years). Given the distribution of inter-arrival times at a processor, for each processor we generate a trace via independent sampling until the target time horizon is reached. The job start time is assumed to be one-year to avoid side-effects related to the synchronous initialization of all nodes/processors. We consider two types of failure traces, namely synthetic and log-based.

Synthetic failure traces - The simulation engine generates a random trace of faults parameterized either by an Exponential fault distribution or by Weibull distribution laws with shape parameter either 0.5 or 0.7. Note that Exponential faults are widely used for theoretical studies, while Weibull faults are representative of the behavior of real-world platforms [18, 19, 20, 21]. For example, Heien et al. 21] have studied the failure distribution for 6 sources of failures (storage devices, NFS, batch system, memory and processor cache errors, etc.), and the aggregate failure distribution. They have shown that the aggregate failure distribution is best modeled by a Weibull distribution with a shape parameter that is between 0.5841 and 0.7097 .

The Jaguar platform, which comprised $N=45,208$ processors, is reported to have experienced about one fault per day [1, which leads to an individual (processor) MTBF $\mu_{\text {ind }}$ equal to $\frac{45,208}{365} \approx 125$ years. Therefore, we set the individual (processor) MTBF to $\mu_{\text {ind }}=125$ years. We let the total number of processors $N$ vary from $N=16,384$ to $N=524,288$, so that the platform MTBF $\mu$ varies from 
$\mu=4,010 \mathrm{~min}$ (about 2.8 days) down to $\mu=125 \mathrm{~min}$ (about 2 hours). Whatever the underlying failure distribution, it is scaled so that its expectation corresponds to the platform MTBF $\mu$. The application size is set to TIME $\mathrm{base}=10,000$ years $/ \mathrm{N}$.

Log-based failure traces - To corroborate the results obtained with synthetic failure traces, and to further assess the performance of our algorithms, we also perform simulations using the failure logs of two production clusters. We use logs of the largest clusters among the preprocessed logs in the Failure trace archive [22, i.e., for clusters at the Los Alamos National Laboratory [19. In these logs, each failure is tagged by the node - and not the processor - on which the failure occurred. Among the 26 possible clusters, we opted for the logs of the only two clusters with more than 1,000 nodes. The motivation is that we need a sample history sufficiently large to simulate platforms with more than ten thousand nodes. The two chosen logs are for clusters 18 (LANL18) and 19 (LANL19) in the archive (referred to as 7 and 8 in [19]). For each $\log$, we record the set $\mathcal{S}$ of availability intervals. The discrete failure distribution for the simulation is generated as follows: the conditional probability $\mathbb{P}(X \geq t \mid X \geq \tau)$ that a node stays up for a duration $t$, knowing that it has been up for a duration $\tau$, is set to the ratio of the number of availability durations in $\mathcal{S}$ greater than or equal to $t$, over the number of availability durations in $\mathcal{S}$ greater than or equal to $\tau$.

The two clusters used for computing our log-based failure distributions consist of 4-processor nodes. Hence, to simulate a platform of, say, $2^{16}$ processors, we generate $2^{14}$ failure traces, one for each 4 processor node. In the logs the individual (processor) MTBF is $\mu_{\text {ind }}=691$ days for the LANL18 cluster, and $\mu_{\text {ind }}=679$ days for the LANL19 cluster. The LANL18 and LANL19 traces are logs for systems which comprised 4,096 processors. Using these logs to generate traces for a system made of 524,288 processors, as the largest platforms we consider with synthetic failure traces, would lead to an obvious risk of oversampling. Therefore, we limit the size of the log-based traces we generate: we let the total number of processors $N$ varies from $N=1,024$ to $N=131,072$, so that the platform MTBF $\mu$ varies from $\mu=971 \mathrm{~min}$ (about 16 hours) down to $\mu=7.5 \mathrm{~min}$. The application size is set to TIME $\mathrm{base}=250$ years/N.

Predicted failures and false predictions - Once we have generated a failure trace, we need to determine which faults are predicted and which are not. In order to do so, we consider all faults in a trace one by one. For each of them, we randomly decide, with probability $r$, whether it is predicted.

We use the simulation engine to generate a random trace of false predictions. The main problem is to decide the shape of the distribution that false predictions should follow. To the best of our knowledge, no published study ever addressed that problem. For synthetic failure traces, we report results when false predictions follow the same distribution than faults (except, of course, that both distributions do not have the same mean value). In B, we report on simulations when false predictions are generated according to a uniform distribution; the results are quite similar. For log-based failures, we only report results when false predictions are generated according to a uniform distribution (because we believe that scaling down a discrete, actual distribution may not be meaningful).

The distribution of false predictions is always scaled so that its expectation is equal to $\frac{\mu_{\mathrm{P}}}{1-p}=\frac{p \mu}{r(1-p)}$, the inter-arrival time of false predictions. Finally, the failure trace and the false-prediction trace are merged to produce the final trace including all events (true predictions, false predictions, and non predicted faults). Each reported value is the average over 100 randomly generated instances.

Checkpointing, recovery, and downtime costs - The experiments use parameters that are representative of current and forthcoming large-scale platforms [23, 24. We take $C=R=10 \mathrm{~min}$, and $D=1$ $\min$ for the synthetic failure traces. For the log-based traces we consider smaller platforms. Therefore, we take $C=R=1 \mathrm{~min}$, and $D=6 \mathrm{~s}$. Whatever the trace, we consider three scenarios for the proactive checkpoints: either proactive checkpoints are (i) exactly as expensive as periodic ones $\left(C_{p}=C\right)$, (ii) ten times cheaper $\left(C_{p}=0.1 C\right)$, and (iii) two times more expensive $\left(C_{p}=2 C\right)$.

Heuristics - In the simulations, we compare four checkpointing strategies:

- RFO is the checkpointing strategy of period $T=\sqrt{2(\mu-(D+R)) C}$ (see Section 3 ).

- OptimalPrediction is the refined algorithm described in Section 4.2 .

- To assess the quality of each strategy, we compare it with its BESTPERIOD counterpart, defined as the same strategy but using the best possible period $T$. This latter period is computed via a bruteforce numerical search for the optimal period (each tested period is evaluated on 100 randomly generated traces, and the period achieving the best average performance is elected as the "best period"). 
Fault predictors - We experiment using the characteristics of two predictors from the literature: one accurate predictor with high recall and precision [7, namely with $p=0.82$ and $r=0.85$, and another predictor with intermediate recall and precision [ $[$, namely with $p=0.4$ and $r=0.7$.

In practice, a predictor will not be able to predict the exact time at which a predicted fault will strike the system. Therefore, in the simulations, when a predictor predicts that a failure will strike the system at a date $t$ (true prediction), the failure actually occurs exactly at time $t$ for heuristic OptimalPrediction, and between time $t$ and time $t+2 C$ for heuristic InexactPrediction (the probability of fault is uniformly distributed in the time-interval). OptimalPrediction can thus be seen as a best case. The comparison between OptimalPrediction and InexactPrediction enables us to assess the impact of the time imprecision of predictions, and to show that the obtained results are quite robust to this type of imprecision. The choice of an interval length of $2 C$ is quite arbitrary. For synthetic traces, this corresponds to $1,200 \mathrm{~s}$, which is quite a significant imprecision.

\subsection{Simulations with synthetic traces}

Figures 3 and 4 show the average waste degradation for the two checkpointing policies, and for their BESTPERIOD counterparts, for both predictors. The waste is reported as a function of the number of processors $N$. We draw the plots as a function of the number of processors $N$ rather than of the platform MTBF $\mu=\mu_{\text {ind }} / N$, because it is more natural to see the waste increase with larger platforms. However, recall that this work is agnostic of the granularity of the processing elements and intrinsically focuses on the impact of the MTBF on the waste.

We also report job execution times, in Table 3 when fault distribution follows an Exponential distribution law, and in Tables 4 and 5 for a Weibull distribution law with shape parameter $k=0.7$ and $k=0.5$ respectively.

Validation of the theoretical study - We used Maple to analytically compute and plot the optimal value of the waste for both the algorithm taking predictions into account, OptimALPREDICTION, and for the algorithm ignoring them, RFO. In order to check the accuracy of our model, we have compared these results with results obtained with the discrete-event simulator.

We first observe that there is a very good correspondence between analytical results and simulations in Figures 3 and 4 . In particular, the Maple plots and the simulations for Exponentially distributed faults are very similar. This shows the validity of the model and of its analysis. Another striking result is that OptimalPrediction has the same waste as its BestPeriod counterpart, even for Weibull fault distributions, in all but the most extreme cases. In the other cases, the waste achieved by OPTIMALPREDICTION is very close to that of its BESTPERIOD counterpart. This demonstrates the very good quality of our checkpointing period $T_{\mathrm{PRED}}$. These conclusions are valid regardless of the cost ratio of periodic and proactive checkpoints.

In Tables 3 through 5 we report the execution times obtained when using the expression of $T$ given by Young 9 and Daly [10 (denoted respectively as Young and DALY) to assess whether $T_{\mathrm{RFO}}$ is a better approximation. (Recall that these three approaches ignore the predictions, which explains why the numbers are identical on both sides of each table.) The expressions of $T$ given by Young, Daly, and RFO are identical for Exponential distributions and the three heuristics achieve the same performance (Table 3). This confirms the analytical evaluation of Table 2 in Section 3 . For Weibull distributions (Tables 4 and 5), RFO achieves lower makespan, and the difference becomes even more significant as the size of the platform increases. Moreover, it is striking to observe in Table 5 that job execution time increases together with the number for processors (from $N=2^{16}$ to $N=2^{19}$ ) if the checkpointing period is DALY or Young. On the contrary, job execution time (rightfully) decreases when using RFO, even if the decrease is moderate with respect to the increase of the platform size. Altogether, the main (striking) conclusion is that RFO should be preferred to both classical approaches for Weibull distributions.

The benefits of prediction - The second observation is that the prediction is useful for the vast majority of the set of parameters under study! In addition, when proactive checkpoints are cheaper than periodic ones, the benefits of fault prediction are increased. On the contrary, when proactive checkpoints are more expensive than periodic ones, the benefits of fault prediction are greatly reduced. One can even observe that the waste with prediction is not better than without prediction in the following scenario: $C_{p}=2 C$, and using the limited-quality predictor $(p=0.4, r=0.7)$ with $2^{19}$ processors, see Figures $4(\mathrm{i}),(\mathrm{j}),(\mathrm{k})$, and $(\mathrm{l})$. 

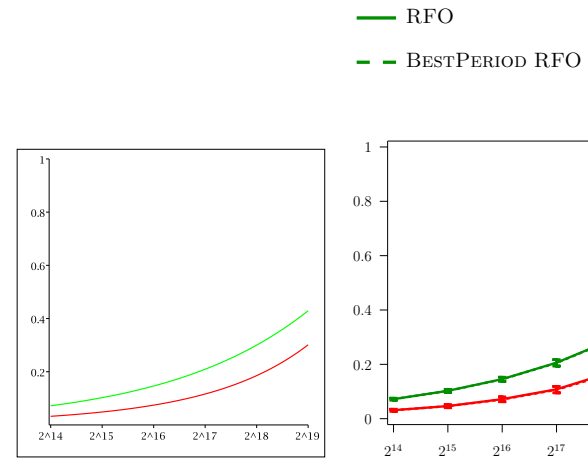

(a) Maple

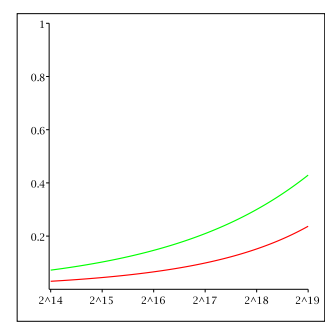

(e) Maple

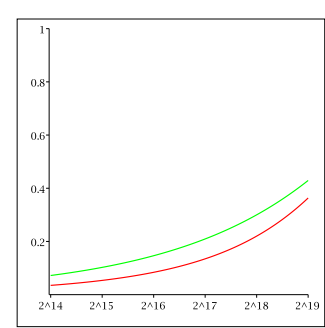

(i) Maple

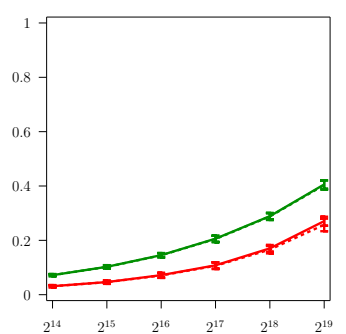

(b) Exponential

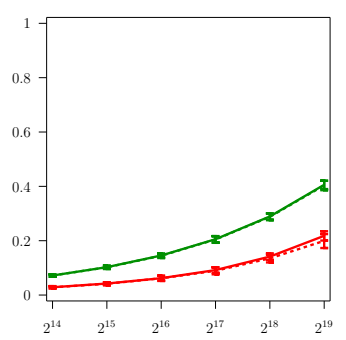

(f) Exponential

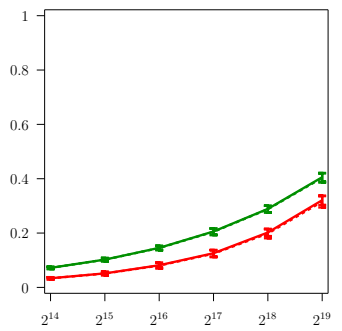

(j) Exponential

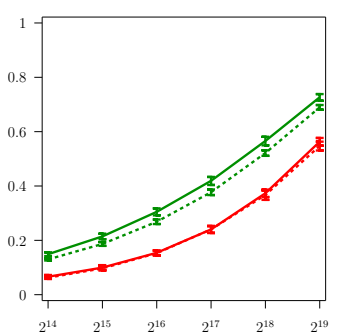

(c) Weibull $k=0.7$

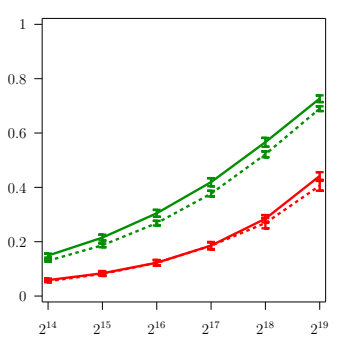

(g) Weibull $k=0.7$

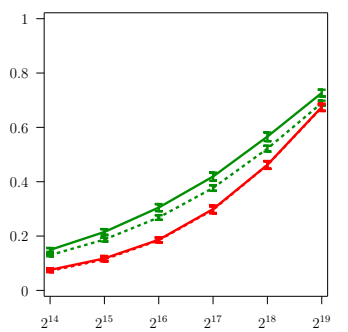

(k) Weibull $k=0.7$

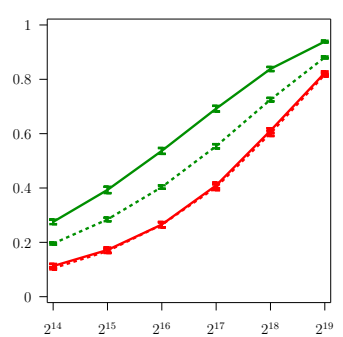

(d) Weibull $k=0.5$

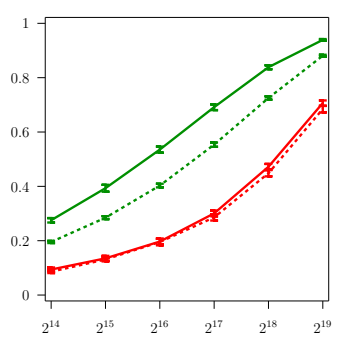

(h) Weibull $k=0.5$

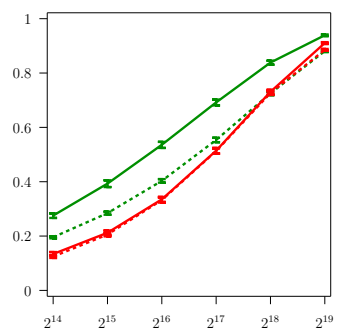

(1) Weibull $k=0.5$

Figure 3: Waste (y-axis) for the different heuristics as a function of the platform size (x-axis), with $p=0.82, r=0.85, C_{p}=C$ (first row), $C_{p}=0.1 C$ (second row), or $C_{p}=2 C$ (third row) and with a trace of false predictions parametrized by a distribution identical to the distribution of the failure trace. 


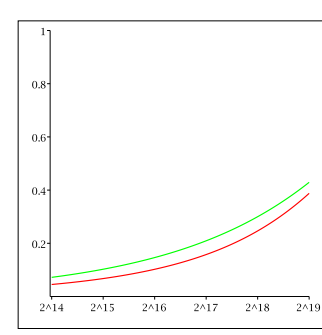

(a) Maple

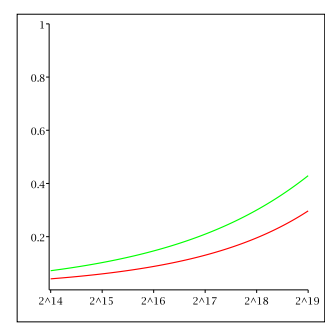

(e) Maple

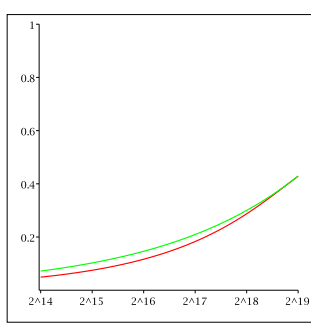

(i) Maple

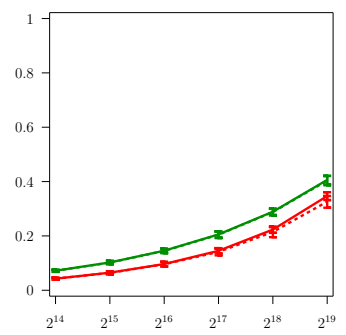

(b) Exponential

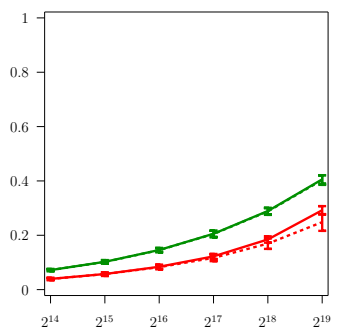

(f) Exponential

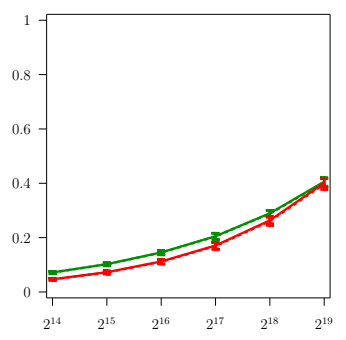

(j) Exponential

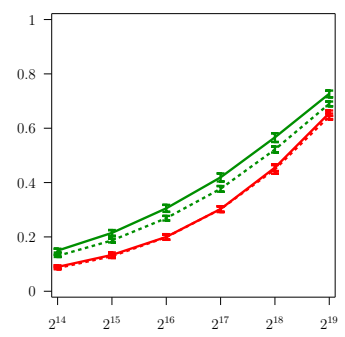

(c) Weibull $k=0.7$

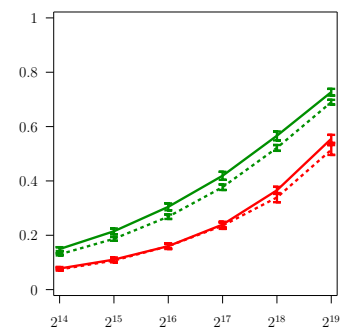

(g) Weibull $k=0.7$

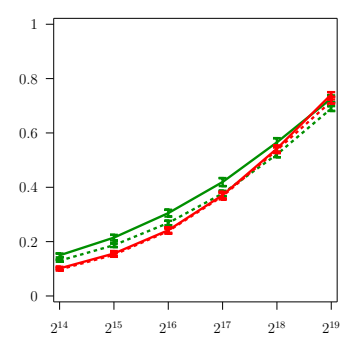

(k) Weibull $k=0.7$

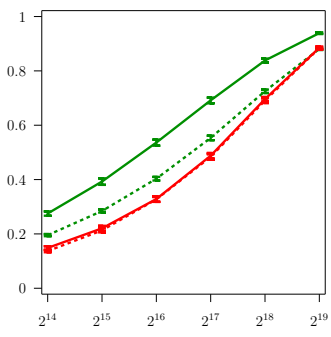

(d) Weibull $k=0.5$

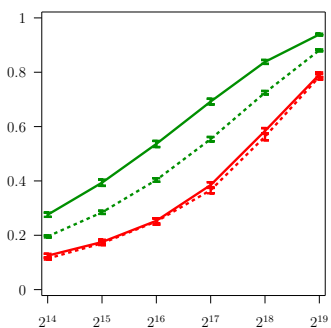

(h) Weibull $k=0.5$

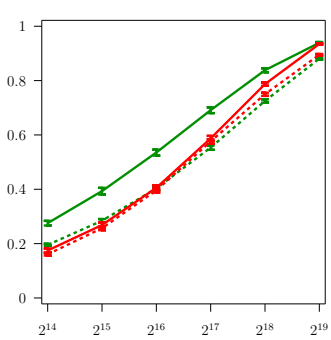

(l) Weibull $k=0.5$

Figure 4: Waste (y-axis) for the different heuristics as a function of the platform size (x-axis), with $p=0.4, r=0.7, C_{p}=C$ (first row), $C_{p}=0.1 C$ (second row), or $C_{p}=2 C$ (third row) and with a trace of false predictions parametrized by a distribution identical to the distribution of the failure trace. 
In Tables 3 through 5 we compute the gain (expressed in percentage) achieved by OptimalPREDICTION over RFO. As a general trend, we observe that the gains due to predictions are more important when the distribution law is further apart from an Exponential distribution. Indeed, the largest gains are when the fault distribution follows a Weibull law of parameter 0.5. Using OptimALPREDICTION in conjunction with a "good" fault predictor we report gains up to $66 \%$ when there is a large number of processors $\left(2^{19}\right)$. The gain is still of $37 \%$ with $2^{16}$ processors. Using a predictor with limited recall and precision, OptimalPREDICTION can still decrease the execution time by $47 \%$ with $2^{19}$ processors, and $31 \%$ with $2^{16}$ processors. In all tested cases, the decrease of the execution times is significant. Gains are less important with Weibull laws of shape parameter $k=0.7$, however they are still reaching a minimum of $13 \%$ with $2^{16}$ processors, and up to $38 \%$ with $2^{19}$ processors. Finally, gains are further reduced with an Exponential law. They are still reaching at least $5 \%$ with $2^{16}$ processors, and up to $19 \%$ with $2^{19}$ processors.

The performance of INEXACTPREDICTION shows that using a fault predictor remains largely beneficial even in the presence of large uncertainties on the time the predicted faults will actually occur (see Tables 3, 4, and 5). When $N=2^{16}$ the degradation with respect to OptimalPREDiCTiON is of $3 \%$ for a Weibull law with shape parameter $k=0.7$, and the minimum gain over RFO is still of $10 \%$. When the shape parameter of the Weibull law is $k=0.5$, the degradation is of $7 \%$ when, for a minimum gain of $26 \%$ over RFO.

\begin{tabular}{c||c|c||c|c||}
\multicolumn{1}{c||}{} & \multicolumn{2}{c||}{\begin{tabular}{c} 
Execution time (in days) \\
\multicolumn{1}{c||}{$C_{p}=C$}
\end{tabular}} & \multicolumn{2}{c||}{$\begin{array}{c}\text { Execution time (in days) } \\
(p=0.82, r=0.85)\end{array}$} \\
& $2^{16}$ procs & $2^{19}$ procs & $2^{16}$ procs & $2^{19}$ procs \\
\hline YOUNG & 65.2 & 11.7 & 65.2 & 11.7 \\
DALY & 65.2 & 11.8 & 65.2 & 11.8 \\
RFO & 65.2 & 11.7 & 65.2 & 11.7 \\
\hline OPTIMALPREDICTION & $60.0(8 \%)$ & $9.5(19 \%)$ & $61.7(5 \%)$ & $10.7(8 \%)$ \\
INEXACTPREDICTION & $60.6(7 \%)$ & $10.2(13 \%)$ & $62.3(4 \%)$ & $11.4(3 \%)$
\end{tabular}

Table 3: Job execution times for an Exponential distribution, and gains due to the fault predictor (with respect to the performance of $\mathrm{RFO})$.

\begin{tabular}{|c|c|c|c|c|}
\hline \multirow[t]{2}{*}{$C_{p}=C$} & \multicolumn{2}{|c|}{$\begin{array}{l}\text { Execution time (in days) } \\
\quad(p=0.82, r=0.85)\end{array}$} & \multicolumn{2}{|c|}{$\begin{array}{l}\text { Execution time (in days) } \\
\qquad(p=0.4, r=0.7)\end{array}$} \\
\hline & $2^{16}$ procs & $2^{19}$ procs & $2^{16}$ procs & $2^{19}$ procs \\
\hline YOUNG & 81.3 & 30.1 & 81.3 & 30.1 \\
\hline DALY & 81.4 & 31.0 & 81.4 & 31.0 \\
\hline $\mathrm{RFO}$ & 80.3 & 25.5 & 80.3 & 25.5 \\
\hline OPTIMALPREDICTION & $65.9(18 \%)$ & $15.9(38 \%)$ & $69.7(13 \%)$ & $20.2(21 \%)$ \\
\hline INEXACTPREDICTION & $68.0(15 \%)$ & $20.3(20 \%)$ & $72.0(10 \%)$ & $24.6(4 \%)$ \\
\hline
\end{tabular}

Table 4: Job execution times for a Weibull distribution with shape parameter $k=0.7$, and gains due to the fault predictor (with respect to the performance of RFO).

\begin{tabular}{c||c|c||c|c||}
\multicolumn{1}{c||}{$C_{p}=C$} & \multicolumn{2}{|c||}{$\begin{array}{c}\text { Execution time (in days) } \\
(p=0.82, r=0.85)\end{array}$} & \multicolumn{2}{c||}{\begin{tabular}{c} 
Execution time (in days) \\
\multicolumn{1}{c||}{$(p=0.4, r=0.7)$}
\end{tabular}} \\
& $2^{16}$ procs & $2^{19}$ procs & $2^{16}$ procs & $2^{19}$ procs \\
\hline YOUNG & 125.5 & 171.8 & 125.5 & 171.8 \\
DALY & 125.8 & 184.7 & 125.8 & 184.7 \\
RFO & 120.2 & 114.8 & 120.2 & 114.8 \\
\hline OPTIMALPREDICTION & $75.9(37 \%)$ & $39.5(66 \%)$ & $83.0(31 \%)$ & $60.8(47 \%)$ \\
INEXACTPREDICTION & $82.0(32 \%)$ & $60.8(47 \%)$ & $89.4(26 \%)$ & $76.6(33 \%)$
\end{tabular}

Table 5: Job execution times for a Weibull distribution with shape parameter $k=0.5$, and gains due to the fault predictor (with respect to the performance of RFO). 


\subsection{Simulations with log-based traces}

Figure 5 shows the average waste degradation for the two checkpointing policies, and for their BESTPERIOD counterparts, for both predictors, both traces, and the three scenarios for proactive checkpoints. Tables 6 and 7 present job execution times for RFO, OptimalPrediction, and InexactPrediction, for both traces and for platform sizes smaller than as the ones reported in Tables 3 through 5 for synthetic traces. The waste for RFO is closer to its BEsTPERIOD counterpart with log-based traces than with Weibull-based traces. As a consequence, when prediction with OPTIMALPREDICTION is beneficial, it is beneficial with respect to both RFO, and to RFO's BESTPERIOD.

Overall, we observe similar results and reach the same conclusions with log-based traces as with synthetic ones. The waste of OptimalPREDICTION is very close to that of its BestPeriod counterpart for platforms containing up to $2^{16}$ processors. This demonstrates the validity of our analysis for the actual traces considered. The waste of OPTIMALPREDICTION is often significantly larger than that of its BESTPERIOD counterpart for platforms containing $2^{17}$ processors. The problem with the largest considered platforms may be due to oversampling. Indeed, the original logs recorded events for platforms comprising only 4,096 processors and respectively contained only 3,010 and 2,343 availability intervals.

As with synthetic failure traces, prediction turns out to be useful for the vast majority of tested configurations. The only cases when prediction is not useful is with the "bad" predictor $(r=0.7$ and $p=0.4)$, when the cost of proactive checkpoint is larger than the cost of periodic checkpoints $\left(C_{p}=2 C\right)$, and when considering the largest of platforms $\left(N=2^{17}\right)$. This extreme case is, however, the only one for which prediction is not beneficial. It is not surprising that predictions are not useful when there are a lot of false predictions that require the use of expensive proactive actions. Looking at Tables 6 and 7. one could remark that performance gains due to the predictions are similar to the ones observed with Exponential-based traces, and are significantly smaller than the ones observed with Weibull-based traces. However, recall that we remarked that gains increase with the size of the platform, and that we consider smaller platforms when using log-based traces.

Finally, the imprecision related to the time where predicted faults strike, induces a performance degradation. However, this degradation is rather limited for the most efficient of the two predictors considered, or when the platform size is not too large.

\begin{tabular}{c||c|c||c|c||}
\multicolumn{1}{c||}{} & \multicolumn{2}{c||}{ Execution time (in days) } & \multicolumn{2}{c||}{ Execution time (in days) } \\
\multicolumn{1}{c||}{$C_{p}=C$} & \multicolumn{2}{c||}{$(p=0.82, r=0.85)$} & \multicolumn{2}{c||}{$(p=0.4, r=0.7)$} \\
& $2^{14}$ procs & $2^{17}$ procs & $2^{14}$ procs & $2^{17}$ procs \\
\hline RFO & 26.8 & 4.88 & 26.8 & 4.88 \\
\hline OPTIMALPREDICTION & $24.4(9 \%)$ & $3.89(20 \%)$ & $25.2(6 \%)$ & $4.44(9 \%)$ \\
INEXACTPREDICTION & $24.7(8 \%)$ & $4.20(14 \%)$ & $25.5(5 \%)$ & $4.73(3 \%)$
\end{tabular}

Table 6: Job execution times with failures based on the failure log of LANL18 cluster, and gains due to the fault predictor (with respect to the performance of RFO).

\begin{tabular}{|c|c|c|c|c|}
\hline \multirow[t]{2}{*}{$C_{p}=C$} & \multicolumn{2}{|c|}{$\begin{array}{l}\text { Execution time (in days) } \\
\quad(p=0.82, r=0.85)\end{array}$} & \multicolumn{2}{|c|}{$\begin{array}{l}\text { Execution time (in days) } \\
\quad(p=0.4, r=0.7)\end{array}$} \\
\hline & $2^{14}$ procs & $2^{17}$ procs & $2^{14}$ procs & $2^{17}$ procs \\
\hline $\mathrm{RFO}$ & 26.8 & 4.86 & 26.8 & 4.86 \\
\hline OPTIMALPREDIC & $24.4(9 \%)$ & $3.85(21 \%)$ & $25.2(6 \%)$ & $4.42(9 \%)$ \\
\hline INEXACTPREDICTION & $24.6(8 \%)$ & $4.14(15 \%)$ & $25.4(5 \%)$ & $4.71(3 \%)$ \\
\hline
\end{tabular}

Table 7: Job execution times with failures based on the failure log of LANL19 cluster, and gains due to the fault predictor (with respect to the performance of RFO).

\subsection{Recall vs. precision}

In this section, we assess the impact of the two key parameters of the predictor, its recall $r$ and its precision $p$. To this purpose, we conduct simulations with synthetic traces, where one parameter is fixed while the other varies. We choose two platforms, a smaller one with $N=2^{16}$ processors (or a MTBF 
(a) $C_{p}=0.1 C$

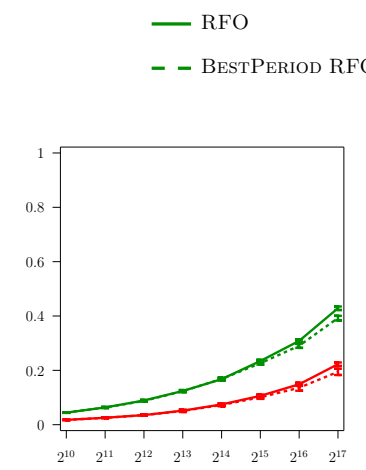

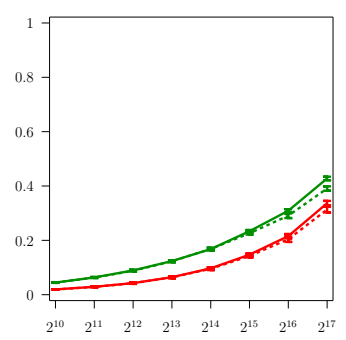

(c) $C_{p}=2 C$

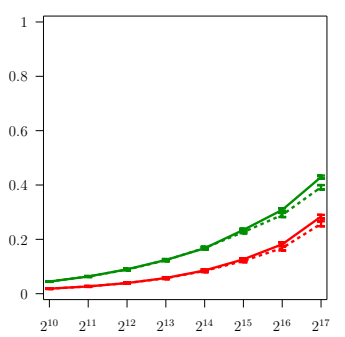

(b) $C_{p}=1 C$

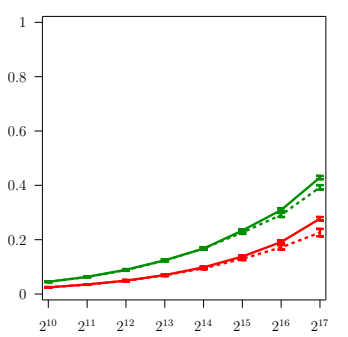

(d) $C_{p}=0.1 C$

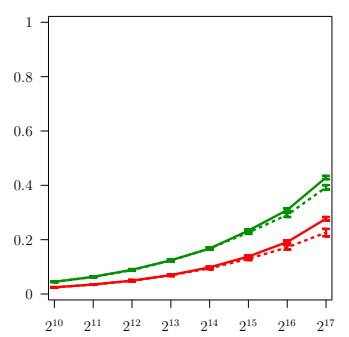

(e) $C_{p}=1 C$

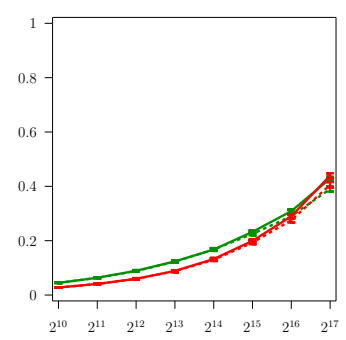

(f) $C_{p}=2 C$

LANL18 cluster with $p=0.4, r=0.7$.

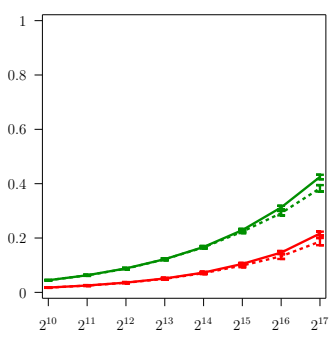

(g) $C_{p}=0.1 C$

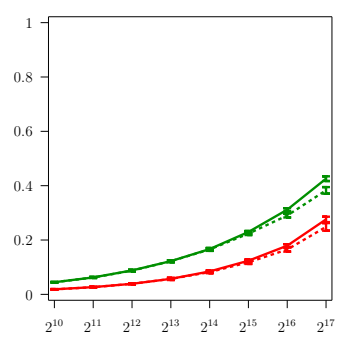

(h) $C_{p}=1 C$

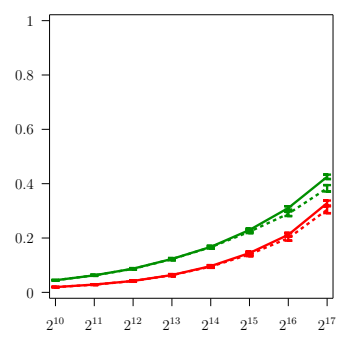

(i) $C_{p}=2 C$

LANL19 cluster with $p=0.82, r=0.85$.

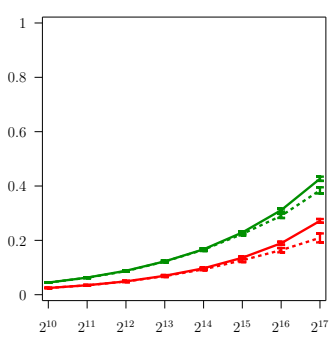

(j) $C_{p}=0.1 C$

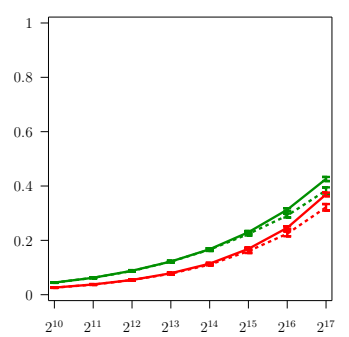

(k) $C_{p}=1 C$

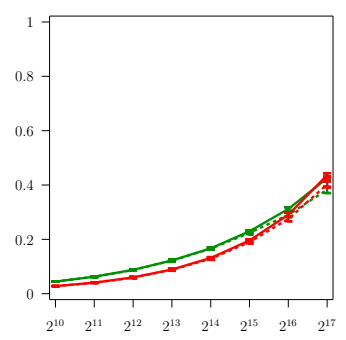

(1) $C_{p}=2 C$

LANL19 cluster with $p=0.4, r=0.7$.

Figure 5: Waste (y-axis) for the different heuristics as a function of the platform size (x-axis) with failures based on the failure log of LANL clusters 18 and 19 . 


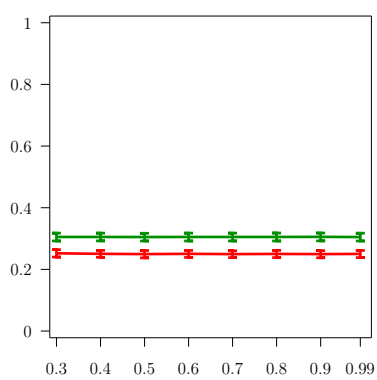

(a) $r=0.4, N=2^{16}$

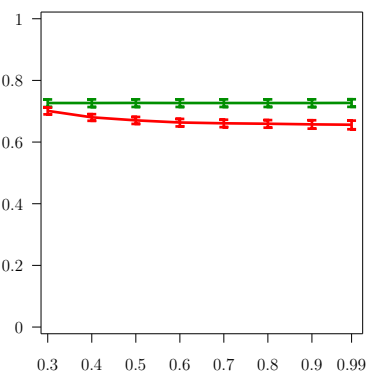

(b) $r=0.4, N=2^{19}$

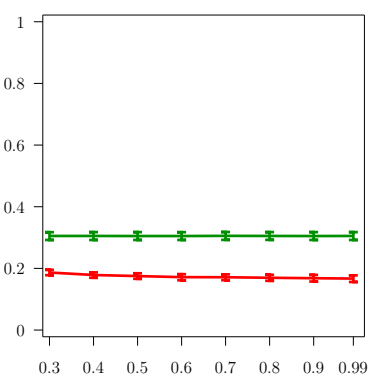

(c) $r=0.8, N=2^{16}$

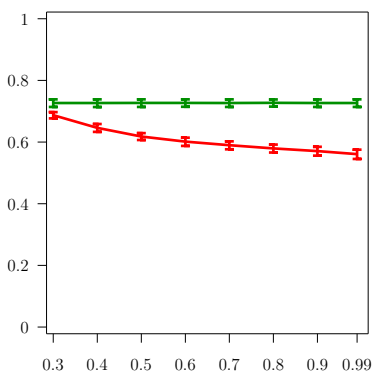

(d) $r=0.8, N=2^{19}$

Figure 6: Waste (y-axis) as a function of the precision (x-axis) for a fixed recall $(r=0.4$ and $r=0.8)$ and for a Weibull distribution of faults (with shape parameter $k=0.7$ ).

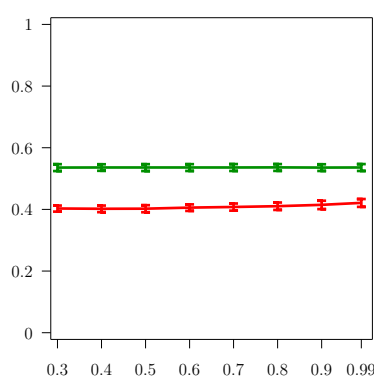

(a) $r=0.4, N=2^{16}$

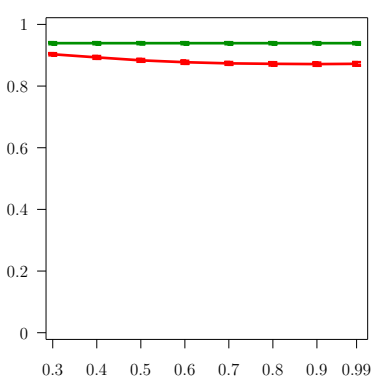

(b) $r=0.4, N=2^{19}$

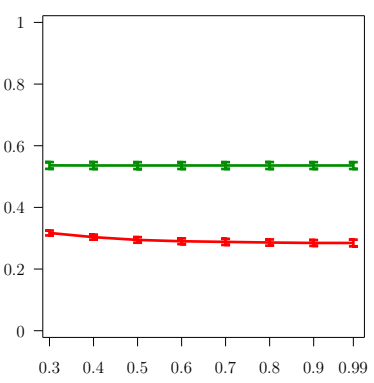

(c) $r=0.8, N=2^{16}$

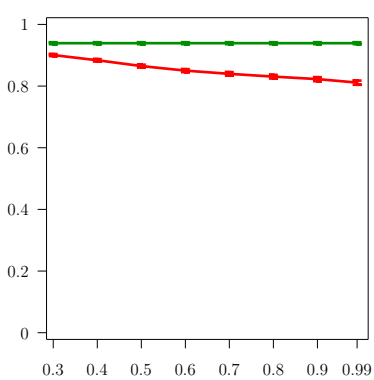

(d) $r=0.8, N=2^{19}$

Figure 7: Waste (y-axis) as a function of the precision (x-axis) for a fixed recall $(r=0.4$ and $r=0.8)$ and for a Weibull distribution of faults (with shape parameter $k=0.5$ ).

$\mu=1,000 \mathrm{~min}$ ) and a larger one with $N=2^{19}$ processors (or a MTBF $\mu=125 \mathrm{~min}$ ). In both cases we study the impact of the predictor characteristics assuming a Weibull fault distribution with shape parameter either 0.5 or 0.7 , under the scenario $C_{p}=C$.

In Figures 6 and 7, we fix the value of $r$ (either $r=0.4$ or $r=0.8$ ) and we let $p$ vary from 0.3 to 0.99 . In the four plots, we observe that the precision has a minor impact on the waste, whether it is with a Weibull distribution of shape parameter 0.7 (Figure 6), or a Weibull distribution of shape parameter 0.5 (Figure 7). In Figures 8 and 9, we conduct the converse experiment and fix the value of $p$ (either $p=0.4$ or $p=0.8$ ), letting $r$ vary from 0.3 to 0.99 . Here we observe that increasing the recall significantly improves performance, in all but one configuration. In the configuration where improving the recall does not make a (significant) difference, there is a very large number of faults and a low precision, hence a large number of false predictions which negatively impact the performance whatever the value of the recall.

Altogether we conclude that it is more important (for the design of future predictors) to focus on improving the recall $r$ rather than the precision $p$, and our results can help quantify this statement. We provide an intuitive explanation as follows: unpredicted faults prove very harmful and heavily increase the waste, while unduly checkpointing due to false predictions (usually) turns out to induce a smaller overhead.

\section{Related work}

Considerable research has been devoted to fault prediction, using very different models (system log analysis [7, event-driven approach [4, 7, 8, support vector machines [6, 3, nearest neighbors [6], etc). In this section we give a brief overview of existing predictors, focusing on their characteristics rather than on the methods of prediction. For the sake of clarity, we sum up the characteristics of the different fault predictors from the literature in Table 8 . 


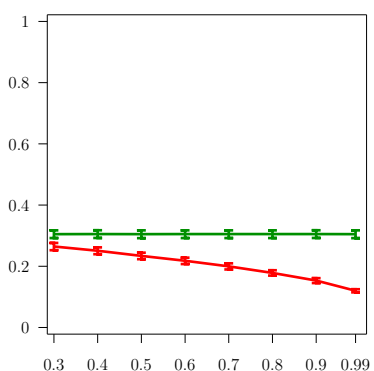

(a) $p=0.4, N=2^{16}$

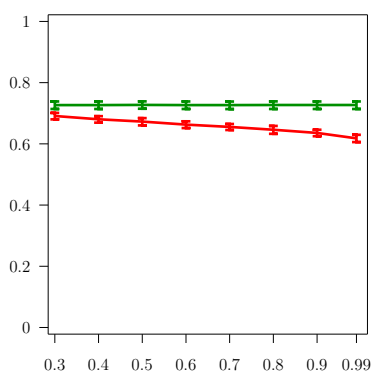

(b) $p=0.4, N=2^{19}$

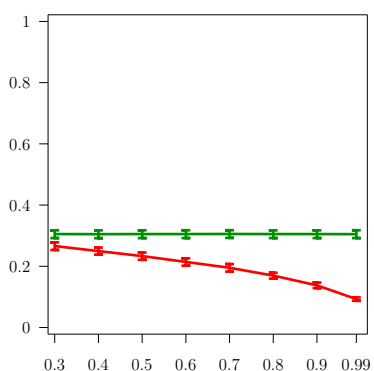

(c) $p=0.8, N=2^{16}$

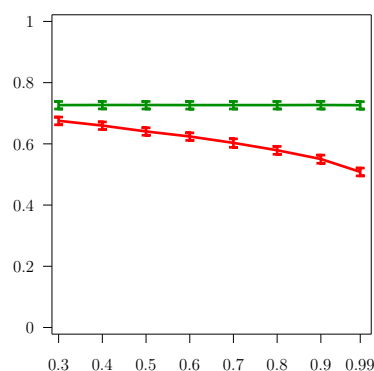

(d) $p=0.8, N=2^{19}$

Figure 8: Waste (y-axis) as a function of the recall (x-axis) for a fixed precision $(p=0.4$ and $p=0.8)$ and for a Weibull distribution $(\mathrm{k}=0.7)$.

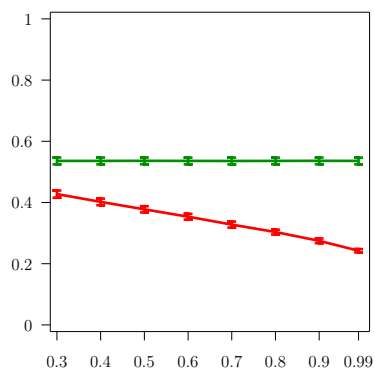

(a) $p=0.4, N=2^{16}$

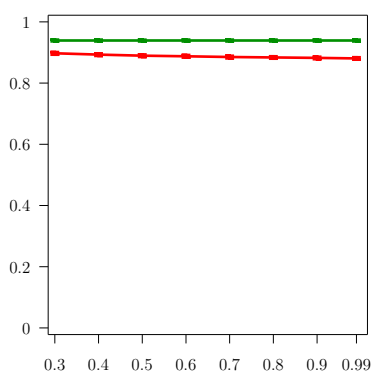

(b) $p=0.4, N=2^{19}$

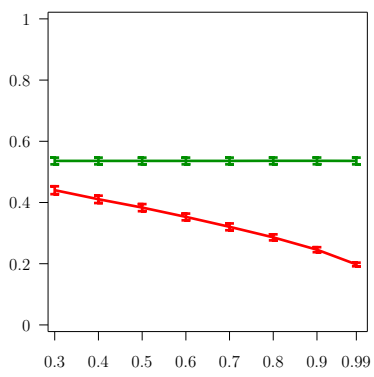

(c) $p=0.8, N=2^{16}$

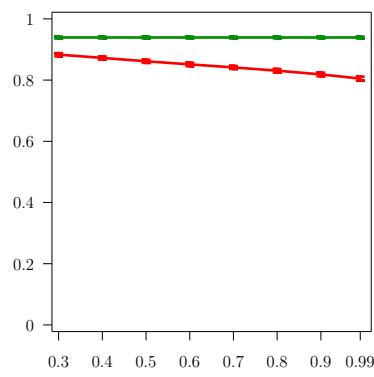

(d) $p=0.8, N=2^{19}$

Figure 9: Waste (y-axis) as a function of the recall (x-axis) for a fixed precision ( $p=0.4$ and $p=0.8$ ) and for a Weibull distribution $(\mathrm{k}=0.5)$.

The authors of [8] introduce the lead time, that is the duration between the time the prediction is made and the time the predicted fault is supposed to happen. This time should be sufficiently large to enable proactive actions. As already mentioned, the distribution of lead times is irrelevant. Indeed, only predictions whose lead time is greater than $C_{p}$, the time to take a proactive checkpoint, are meaningful. Predictions whose lead time is smaller than $C_{p}$, whenever they materialize as actual faults, should be classified as unpredicted faults; the predictor recall should be decreased accordingly.

The predictor of 8 is also able to locate where the predicted fault is supposed to strike. This additional characteristics has a negative impact on the precision (because a fault happening at the predicted time but not on the predicted location is classified as a non predicted fault; see the low value of $p$ in Table 8). The authors of 8 state that fault localization has a positive impact on proactive checkpointing time in their context: instead of a full checkpoint costing 1,500 seconds they can take a partial checkpoint costing only 12 seconds. This led us to introduce a different $\operatorname{cost} C_{p}$ for proactive checkpoints, that can be smaller than the cost $C$ of regular checkpoints. Gainaru et al. [5] also stated that fault-localization could help decrease the checkpointing time. Their predictor also gives information on fault localization. They studied the impact of different lead times on the recall of their predictor. Papers [7] and [6] also considered lead times.

Most studies on fault prediction state that a proactive action must be taken right before the predicted fault, be it a checkpoint or a migration. However, we have shown in this paper that it is beneficial to ignore some predictions, namely when the predicted fault is announced to strike less than $\frac{C_{p}}{p}$ seconds after the last periodic checkpoint.

Gainaru et al. [5] studied the impact of prediction on the checkpointing period. Their computation of the total waste is not fully accurate and they do not provide any minimization analysis. Instead, they only propose to use Young's formula, replacing the MTBF by the mean-time of unpredicted faults. They do not question whether all predictions should be taken into account. Furthermore, they did not conduct any simulations; instead they analytically computed the ratio of the waste with and without predictions and instantiated the corresponding formula with several scenarios. 


\begin{tabular}{|c|c|c|c|}
\hline Paper & Lead Time & Precision & Recall \\
\hline$[8]$ & $300 \mathrm{~s}$ & $40 \%$ & $70 \%$ \\
{$[8]$} & $600 \mathrm{~s}$ & $35 \%$ & $60 \%$ \\
{$[7]$} & $2 \mathrm{~h}$ & $64.8 \%$ & $65.2 \%$ \\
{$[7]$} & $0 \mathrm{~min}$ & $82.3 \%$ & $85.4 \%$ \\
{$[4]$} & $32 \mathrm{~s}$ & $93 \%$ & $43 \%$ \\
{$[5]$} & $10 \mathrm{~s}$ & $92 \%$ & $40 \%$ \\
{$[5]$} & $60 \mathrm{~s}$ & $92 \%$ & $20 \%$ \\
{$[5]$} & $600 \mathrm{~s}$ & $92 \%$ & $3 \%$ \\
{$[3$} & $\mathrm{NA}$ & $70 \%$ & $75 \%$ \\
{$[6]$} & $\mathrm{NA}$ & $20 \%$ & $30 \%$ \\
{$[6]$} & $\mathrm{NA}$ & $30 \%$ & $75 \%$ \\
{$[6]$} & $\mathrm{NA}$ & $40 \%$ & $90 \%$ \\
{$[6]$} & $\mathrm{NA}$ & $50 \%$ & $30 \%$ \\
{$[6]$} & $\mathrm{NA}$ & $60 \%$ & $85 \%$ \\
\hline
\end{tabular}

Table 8: Comparative study of different parameters returned by some predictors.

Li et al. 25] considered the mathematical problem of when and how to migrate. In order to be able to use migration, they assumed that at any time $2 \%$ of the resources are available as spares. This allows them to conceive a Knapsack-based heuristic. Thanks to their algorithm, they were able to save $30 \%$ of the execution time compared to a heuristic that does not take the prediction into account, with a precision and recall of $70 \%$, and with a maximum load of 0.7 . In our study we do not consider that we have a batch of spare resources. We assume that after a downtime the resources that failed are once again available.

Note that some authors [7, 6] do not consider that their predictors predict the exact time of the fault. On the contrary, they consider a "prediction window" which is the time interval in which the predicted is supposed to occur. Because most papers focus on prediction windows of negligible length, we did not consider prediction windows in this study.

Finally, to the best of our knowledge, this work is the first to focus on the mathematical aspect of fault prediction, and to provide a model and a detailed analysis of the waste due to all three types of events (true and false predictions and unpredicted failures).

\section{Conclusion}

In this work we have studied the impact of fault prediction on periodic checkpointing. We started by revisiting the first-order approach by Young and Daly. We have performed a refined analysis leading to a better checkpointing period: $T_{\mathrm{RFO}}$ is slightly closer to the optimal period for Exponential distributions (the only case where the optimal is known), and leads to smaller execution times for Weibull distributions (as shown in Section 5.2).

Then we have extended the analysis to include fault predictions. We have established analytical conditions stating whether a fault prediction should be taken into account or not. More importantly, we have proven that the optimal approach is to never trust the predictor in the beginning of a regular period, and to always trust it in the end of the period; the cross-over point $\frac{C_{p}}{p}$ depends on the time to take a proactive checkpoint and on the precision of the predictor. This striking result is somewhat unexpected, as one might have envisioned more trust regimes, with several intermediate trust levels smoothly evolving from a "never trust" policy to an "always trust" one.

We have conducted simulations involving synthetic failure traces following either an Exponential distribution law or a Weibull one. We have also used log-based failure traces. In addition, we have used exact prediction dates and uncertainty intervals for these dates. Through this extensive experiment setting, we have established the accuracy of the model, of its analysis, and of the predicted period (in the presence of a fault predictor). The simulations also show that even a not-so-good fault predictor can lead to quite a significant decrease in the application execution time. We have also shown that the most important characteristic of a fault predictor is its recall (the percentage of actually predicted faults) 
rather than its precision (the percentage of predictions that actually correspond to faults): better safe than sorry, or better prepare for a false event than miss an actual failure!

Altogether, the analytical model and the comprehensive results provided in this work enable to fully assess the impact of fault prediction on optimal checkpointing strategies. Future work will be devoted to the study of the impact of fault prediction on uncoordinated or hierarchical checkpointing protocols. Another challenging problem is to determine the best trade-off between performance and energy consumption when combining several resilience techniques such as checkpointing, prediction, and replication.

\section{Acknowledgments.}

Y. Robert is with the Institut Universitaire de France. This work was supported in part by the ANR RESCUE project. We would like to thank the reviewers of JPDC for their comments and suggestions, which greatly helped improve the final version of the paper.

\section{References}

[1] G. Zheng, X. Ni, L. Kale, A scalable double in-memory checkpoint and restart scheme towards exascale, in: Dependable Systems and Networks Workshops (DSN-W), 2012. doi:10.1109/DSNW. 2012.6264677 .

[2] K. Sato, A. Moody, K. Mohror, T. Gamblin, B. R. de Supinski, N. Maruyama, S. Matsuoka, Design and modeling of a non-blocking checkpointing system, in: SC'12 (the 2012 International Conference for High Performance Computing, Networking, Storage and Analysis), 2012.

[3] E. W. Fulp, G. A. Fink, J. N. Haack, Predicting computer system failures using support vector machines, in: Proceedings of the First USENIX conference on Analysis of system logs, USENIX Association, 2008.

[4] A. Gainaru, F. Cappello, W. Kramer, Taming of the shrew: Modeling the normal and faulty behavior of large-scale hpc systems, in: Proc. IPDPS'12, 2012.

[5] A. Gainaru, F. Cappello, W. Kramer, M. Snir, Fault prediction under the microscope - a closer look into hpc systems, in: SC'12 (the 2012 International Conference for High Performance Computing, Networking, Storage and Analysis), 2012.

[6] Y. Liang, Y. Zhang, H. Xiong, R. K. Sahoo, Failure prediction in ibm bluegene/l event logs, in: ICDM, 2007, pp. 583-588.

[7] L. Yu, Z. Zheng, Z. Lan, S. Coghlan, Practical online failure prediction for blue gene/p: Period-based vs event-driven, in: Dependable Systems and Networks Workshops (DSN-W), 2011, pp. 259-264. doi:10.1109/DSNW.2011.5958823.

[8] Z. Zheng, Z. Lan, R. Gupta, S. Coghlan, P. Beckman, A practical failure prediction with location and lead time for blue gene/p, in: Dependable Systems and Networks Workshops (DSN-W), 2010, pp. 15-22. doi:10.1109/DSNW. 2010.5542627.

[9] J. W. Young, A first order approximation to the optimum checkpoint interval, Comm. of the ACM 17 (9) (1974) 530-531.

[10] J. T. Daly, A higher order estimate of the optimum checkpoint interval for restart dumps, FGCS 22 (3) (2004) 303-312.

[11] N. Kolettis, N. D. Fulton, Software rejuvenation: Analysis, module and applications, in: FTCS '95, IEEE CS, Washington, DC, USA, 1995, p. 381.

[12] V. Castelli, R. E. Harper, P. Heidelberger, S. W. Hunter, K. S. Trivedi, K. Vaidyanathan, W. P. Zeggert, Proactive management of software aging, IBM J. Res. Dev. 45 (2) (2001) 311-332. 
[13] J. Hong, S. Kim, Y. Cho, H. Yeom, T. Park, On the choice of checkpoint interval using memory usage profile and adaptive time series analysis, in: Proc. Pacific Rim Int. Symp. on Dependable Computing, IEEE Computer Society, 2001.

[14] J. Wingstrom, Overcoming The Difficulties Created By The Volatile Nature Of Desktop Grids Through Understanding, Prediction And Redundancy, Ph.D. thesis, University of Hawai'i at Manoa (2009).

[15] M. Bougeret, H. Casanova, M. Rabie, Y. Robert, F. Vivien, Checkpointing strategies for parallel jobs, in: Proceedings of SC'11, 2011.

[16] Y. Robert, F. Vivien, D. Zaidouni, On the complexity of scheduling checkpoints for computational workflowss, in: FTXS'2012, the Workshop on Fault-Tolerance for HPC at Extreme Scale, in conjunction with the 42nd Annual IEEE/IFIP Int. Conf. on Dependable Systems and Networks (DSN 2012), IEEE Computer Society Press, 2012.

[17] M. Mitzenmacher, E. Upfal, Probability and Computing: Randomized Algorithms and Probabilistic Analysis, Cambridge University Press, 2005.

[18] T. Heath, R. P. Martin, T. D. Nguyen, Improving cluster availability using workstation validation, SIGMETRICS Perf. Eval. Rev. 30 (1).

[19] B. Schroeder, G. A. Gibson, A large-scale study of failures in high-performance computing systems, in: Proc. of DSN, 2006, pp. 249-258.

[20] Y. Liu, R. Nassar, C. Leangsuksun, N. Naksinehaboon, M. Paun, S. Scott, An optimal checkpoint/restart model for a large scale high performance computing system, in: IPDPS'08, IEEE, 2008.

[21] E. Heien, D. Kondo, A. Gainaru, D. LaPine, B. Kramer, F. Cappello, Modeling and tolerating heterogeneous failures in large parallel systems, in: Proc. ACM/IEEE Supercomputing'11, ACM Press, 2011.

[22] D. Kondo, B. Javadi, A. Iosup, D. Epema, The failure trace archive: Enabling comparative analysis of failures in diverse distributed systems, Cluster Computing and the Grid, IEEE International Symposium on 0 (2010) 398-407. doi:http://doi.ieeecomputersociety.org/10.1109/CCGRID. 2010.71 .

[23] F. Cappello, H. Casanova, Y. Robert, Preventive migration vs. preventive checkpointing for extreme scale supercomputers, Parallel Processing Letters 21 (2) (2011) 111-132.

[24] K. Ferreira, J. Stearley, J. H. I. Laros, R. Oldfield, K. Pedretti, R. Brightwell, R. Riesen, P. G. Bridges, D. Arnold, Evaluating the Viability of Process Replication Reliability for Exascale Systems, in: Proceedings of the 2011 ACM/IEEE Conf. on Supercomputing, 2011.

[25] Y. Li, Z. Lan, P. Gujrati, X. Sun, Fault-aware runtime strategies for high-performance computing, Parallel and Distributed Systems, IEEE Transactions on 20 (4) (2009) 460-473.

[26] S. M. Ross, Introduction to Probability Models, Tenth Edition, Academic Press, 2009.

\section{A}

For the sake of completeness, we provide a proof of the following result:

Proposition 2. Consider a platform comprising $N$ components, and assume that the inter-arrival times of the faults on the components are independent and identically distributed random variables that follow an arbitrary probability law whose expectation is $\mu_{i n d}$. Then the expectation of the inter-arrival times of the faults on the whole platform is $\mu=\frac{\mu_{\text {ind }}}{N}$. 
Proof. Consider first a single component, say component number $q$. Let $X_{i}, i \geq 0$ denote the IID random variables for fault inter-arrival times on that component, with $\mathbb{E}\left(X_{i}\right)=\mu_{\text {ind }}$. Consider a fixed time bound $F$. Let $n_{q}(F)$ be the number of faults on the component until time $F$ is exceeded. In other words, the $\left(n_{q}(F)-1\right)$-th fault is the last one to happen strictly before time $F$, and the $n_{q}(F)$-th fault is the first to happen at time $F$ or after. By definition of $n_{q}(F)$, we have

$$
\sum_{i=1}^{n_{q}(F)-1} X_{i} \leq F \leq \sum_{i=1}^{n_{q}(F)} X_{i}
$$

Using Wald's equation [26, p. 486], with $n_{q}(F)$ as a stopping criterion, we derive:

$$
\left(\mathbb{E}\left(n_{q}(F)\right)-1\right) \mu_{\text {ind }} \leq F \leq \mathbb{E}\left(n_{q}(F)\right) \mu_{\text {ind }}
$$

and we obtain:

$$
\lim _{F \rightarrow+\infty} \frac{\mathbb{E}\left(n_{q}(F)\right)}{F}=\frac{1}{\mu_{\text {ind }}}
$$

Consider now the whole platform, and let $Y_{i}, i \geq 0$ denote the IID random variables for fault interarrival times on the platform, with $\mathbb{E}\left(Y_{i}\right)=\mu$. Consider a fixed time bound $F$ as before. Let $n(F)$ be the number of faults on the whole platform until time $F$ is exceeded. With the same reasoning for the whole platform as for a single component, we derive:

$$
\lim _{F \rightarrow+\infty} \frac{\mathbb{E}(n(F))}{F}=\frac{1}{\mu}
$$

Now let $m_{q}(F)$ be the number of these faults that strike component number $q$. Of course we have $n(F)=\sum_{q=1}^{N} m_{q}(F)$. By definition, except for the component hit by the last failure, $m_{q}(F)+1$ is the number of failures on component $q$ until time $F$ is exceeded, hence $n_{q}(F)=m_{q}(F)+1$ (and this number is $m_{q}(F)=n_{q}(F)$ on the component hit by the last failure). From Equation (18) again, we have for each component $q$ :

$$
\lim _{F \rightarrow+\infty} \frac{\mathbb{E}\left(m_{q}(F)\right)}{F}=\frac{1}{\mu_{\text {ind }}}
$$

Since $n(F)=\sum_{q=1}^{N} m_{q}(F)$, we also have:

$$
\lim _{F \rightarrow+\infty} \frac{\mathbb{E}(n(F))}{F}=\frac{N}{\mu_{\text {ind }}}
$$

Equations 190 and $20 a d$ to the result.

\section{B}

In this section, we provide results for synthetic failure traces when false predictions are generated according to a uniform distribution. 

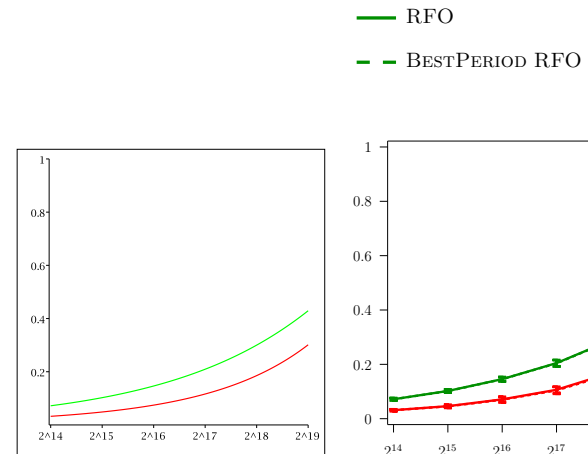

(a) Maple

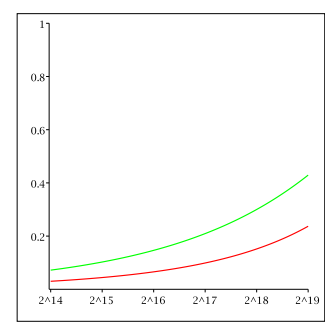

(e) Maple

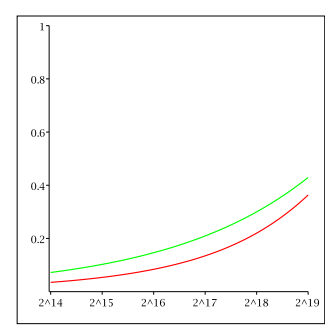

(i) Maple

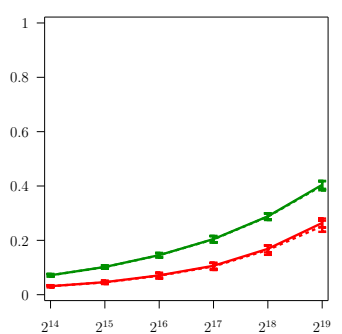

(b) Exponential

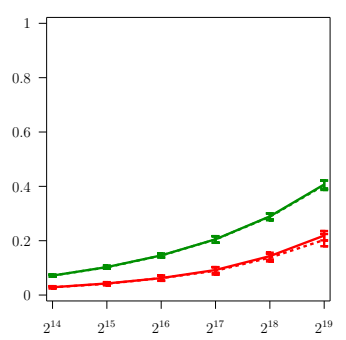

(f) Exponential

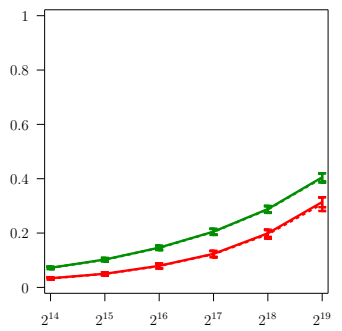

(j) Exponential

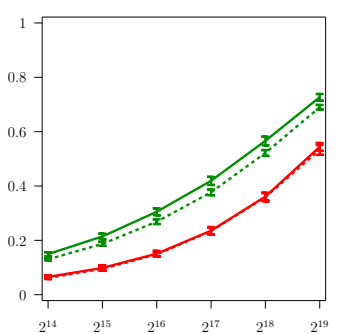

(c) Weibull $k=0.7$

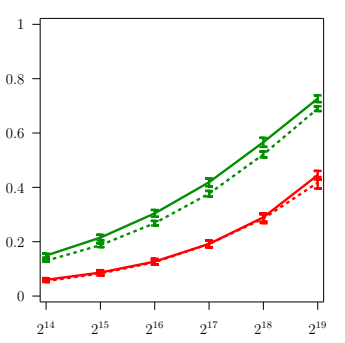

(g) Weibull $k=0.7$

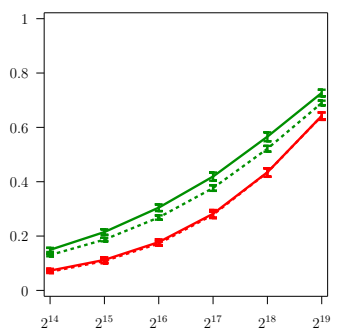

(k) Weibull $k=0.7$

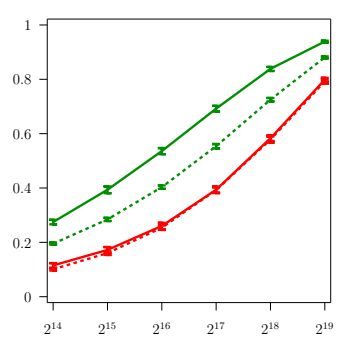

(d) Weibull $k=0.5$

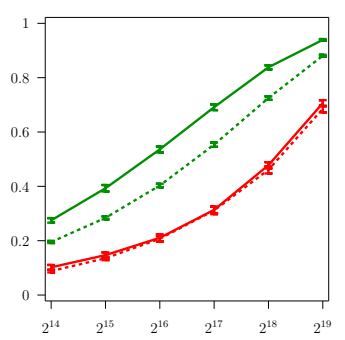

(h) Weibull $k=0.5$

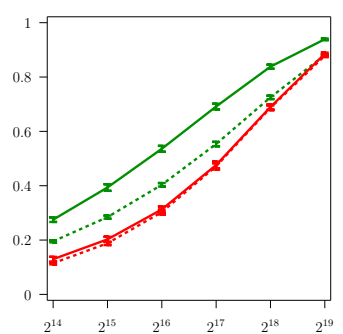

(l) Weibull $k=0.5$

Figure 10: Waste (y-axis) for the different heuristics as a function of the platform size (x-axis), with $p=0.82, r=0.85, C_{p}=C$ (first row), $C_{p}=0.1 C$ (second row), or $C_{p}=2 C$ (third row) and with a trace of false predictions parametrized by a uniform distribution. 


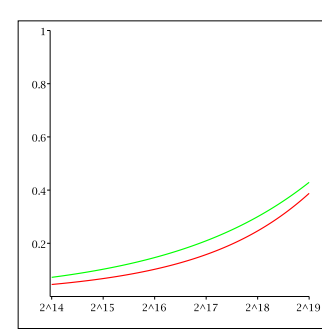

(a) Maple

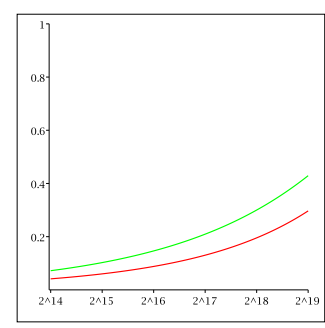

(e) Maple

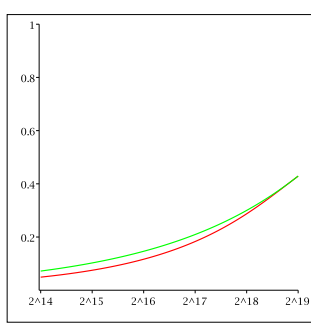

(i) Maple

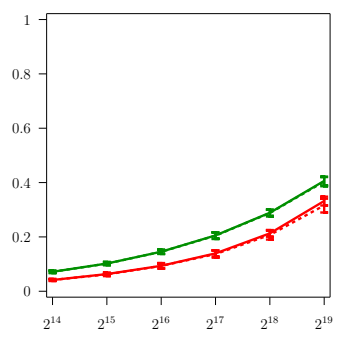

(b) Exponential

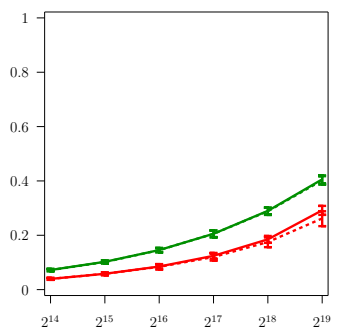

(f) Exponential

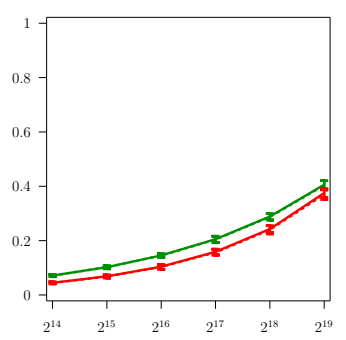

(j) Exponential

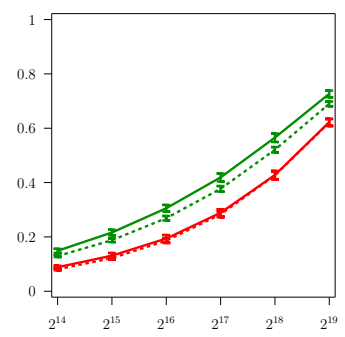

(c) Weibull $k=0.7$

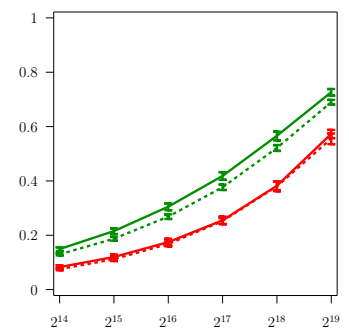

(g) Weibull $k=0.7$

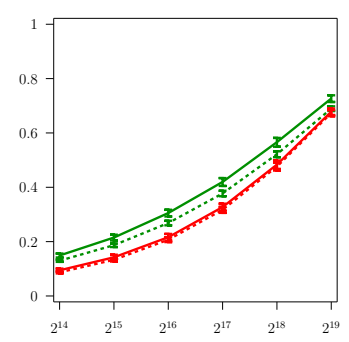

(k) Weibull $k=0.7$

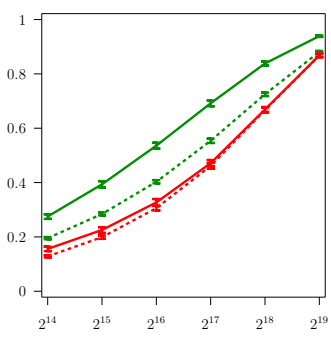

(d) Weibull $k=0.5$

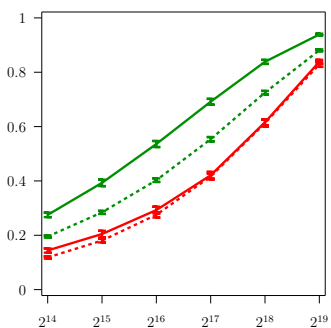

(h) Weibull $k=0.5$

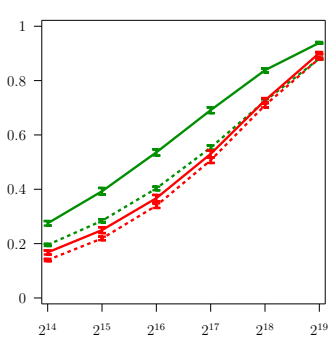

(1) Weibull $k=0.5$

Figure 11: Waste (y-axis) for the different heuristics as a function of the platform size (x-axis), with $p=0.4, r=0.7, C_{p}=C$ (first row), $C_{p}=0.1 C$ (second row), or $C_{p}=2 C$ (third row) and with a trace of false predictions parametrized by a uniform distribution.. 